Article

\title{
Synthesis, Characterization, Antimicrobial Screening and Free-Radical Scavenging Activity of Some Novel Substituted Pyrazoles
}

\author{
Nagwa Mohamed Mahrous Hamada * and Nadia Yousef Megally Abdo \\ Department of Chemistry, Faculty of Education, Alexandria University, Alexandria 21526, Egypt; \\ E-Mail: nadiamegally@yahoo.com \\ * Author to whom correspondence should be addressed; E-Mail: nagwahamada2002@yahoo.com or \\ nagwahamada2002@alex.edu.eg; Tel.: +20-106-560-6949; Fax: +20-3-590-1516.
}

Academic Editor: Philippe Belmont

Received: 26 April 2015 / Accepted: 1 June 2015 / Published: 8 June 2015

\begin{abstract}
The present work deals with the synthesis of acetoxysulfonamide pyrazole derivatives, substituted 4,5-dihydropyrazole-1-carbothioamide and 4,5-dihydropyrazole-1isonicotinoyl derivatives starting from substituted vanillin chalcones. Acetoxysulfonamide pyrazole derivatives were prepared from the reaction of chalcones with $p$-sulfamylphenylhydrazine followed by treatment with acetic anhydride. At the same time 4,5-dihydropyrazole-1-carbothioamide and 4,5-dihydropyrazole-1-isonicotinoyl derivatives were prepared from the reaction of chalcones with either thiosemicarbazide or isonicotinic acid hydrazide, respectively. The synthesized compounds were structurally characterized on the basis of IR, ${ }^{1} \mathrm{H}-\mathrm{NMR},{ }^{13} \mathrm{C}-\mathrm{NMR}$ spectral data and microanalyses. All of the newly isolated compounds were tested for their antimicrobial activities. The antimicrobial screening using the agar well-diffusion method revealed that the chloro derivatives are the most active ones. Moreover, the antioxidant and anti-inflammatory activity of these chloro derivatives are also studied using the DPPH radical scavenging and NO radical scavenging methods, respectively.
\end{abstract}

Keywords: chalcones; sulfonamide pyrazole; isonicotinic acid hydrazide; antimicrobial activity; antioxidant; anti-inflammatory 


\section{Introduction}

Pyrazole and its derivatives represent one of the most active classes of heterocyclic compounds possessing a wide spectrum of biological activities. In particular, they are used as antitumor [1], antibacterial, antifungal, antiviral, antiparasitic, antitubercular, insecticidal, anti-inflammatory, antidiabetic and analgesic compounds [2-14]. Pyrazoles are also used extensively as useful synthons in organic synthesis [15-22]. A literature survey reveals that a significant portion of research in heterocyclic chemistry has been devoted to pyrazoles containing different aryl groups as substituents [23-30]. For example, celecoxib (Figure 1) is a sulfonamide non-steroidal anti-inflammatory drug [31]. The structure activity relationships of celecoxib attracted our attention and prompted us to synthesize some matching pyrazole derivatives with some structural modifications.

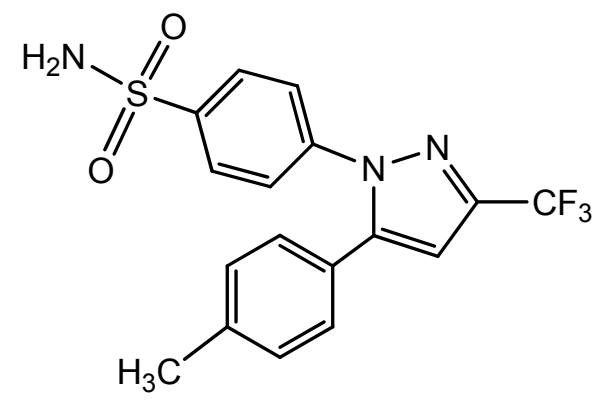

Figure 1. Structural formula of celecoxib.

Similarly, there has been a considerable interest in the chemistry of the 2-pyrazoline ring system which is a core structure in various synthetic pharmaceuticals with a broad spectrum of biological activities. Their pharmaceutical importance lies in the fact that they can be effectively utilized as antibacterial, potential antimicrobial [32], anti-inflammatory [33], analgesic [34], antidepressant [35-37], anticancer [38], antiproteolytic [39], antiviral [40], antihypertensive [41], antitubercular [42], and anticonvulsant [43] compounds. As a part of our program aiming at the synthesis of different heterocyclic derivatives, we report here in the convenient synthesis of some new pyrazoles $\mathbf{3 a}-\mathbf{e}$ and dihydropyrazoles $\mathbf{4 a - e}$ and $\mathbf{5 a}-\mathbf{e}$ starting from chalcones 1a-e. which exhibit efficient antimicrobial, antioxidant and anti-inflammatory activities.

\section{Results and Discussion}

\subsection{Chemistry}

The synthesis of chalcones 1a-e was accomplished by a one-pot Claisen-Schmidt condensation [44,45] in $60 \%$ ethanol between the appropriate acetophenone derivative and 4-hydroxy-3-methoxybenzaldehyde (vanillin). In all the synthesized chalcones, only the trans double bond was obtained (on the basis of the corresponding NMR coupling constant). All synthesized compounds were characterized by spectral data (IR, ${ }^{1} \mathrm{H}-\mathrm{NMR}$ and ${ }^{13} \mathrm{C}-\mathrm{NMR}$ ) that was consistent with the proposed structures. The IR spectra of 1a-e revealed the characteristic bands for $\mathrm{C}=\mathrm{O}$ at $1661-1684$, vinyl $\mathrm{CH}=\mathrm{CH}$ that appeared at $1593-1618$ and $\mathrm{OH}$ at $3424-3441 \mathrm{~cm}^{-1}$. The ${ }^{1} \mathrm{H}-\mathrm{NMR}$ spectra showed the presence of a broad singlet at $\delta=11.22-11.72 \mathrm{ppm}$ for the phenolic $\mathrm{OH}$ proton, multiplets at $\delta=7.30-7.97$ characteristic 
of the aromatic protons, a doublet $(J=15 \mathrm{~Hz})$ at $\delta=7.22-7.29 \mathrm{ppm}$ characteristic of the olefinic $\mathrm{COCH}=\mathrm{CH}$, another doublet $(J=15 \mathrm{~Hz})$ at $\delta=6.61-6.89 \mathrm{ppm}$ characteristic of the olefinic $\mathrm{COCH}=\mathrm{C} H$. The methyl protons appeared as a singlet in the $\delta=3.21-3.36 \mathrm{ppm}$ range.

The hydrazone derivatives $\mathbf{2 a}-\mathbf{e}$ were obtained by treatment of $\mathbf{1 a}-\mathbf{e}$ with $p$-sulphamylphenyl- hydrazine in glacial acetic acid. The IR spectra of $\mathbf{2 a}-\mathbf{e}$ showed the characteristic bands for a vinyl $\mathrm{CH}=\mathrm{CH}$ group at $1603-1619 \mathrm{~cm}^{-1}$, the phenolic $\mathrm{OH}$ in the $3430-3441 \mathrm{~cm}^{-1}$ range and a primary or secondary amine band at 3379-3391 and 3310-3350 $\mathrm{cm}^{-1}$, respectively. The ${ }^{1} \mathrm{H}-\mathrm{NMR}$ spectra showed the presence of a singlet at $\delta=11.22-11.39 \mathrm{ppm}$ for the $\mathrm{OH}$ proton, a singlet equivalent to one proton in the $\delta=8.21-8.73 \mathrm{ppm}$ range characteristic of a hydrazone $\mathrm{NH}$ proton, while the primary amine $\mathrm{NH}_{2}$ protons appeared at $\delta=9.23-9.40 \mathrm{ppm}$. A multiplet at $\delta=7.26-7.82 \mathrm{ppm}$ is characteristic of the aromatic protons, while a doublet at $\delta=7.53-7.61 \mathrm{ppm}$ for $\mathrm{N}=\mathrm{C}-\mathrm{CH}=\mathrm{CH}(J=13 \mathrm{~Hz})$, another doublet at $\delta=6.69-6.85 \mathrm{ppm}(J=13 \mathrm{~Hz})$ for $\mathrm{N}=\mathrm{C}-\mathrm{CH}=\mathrm{CH}$ and a singlet equivalent to three protons at $\delta=3.19-3.37 \mathrm{ppm}$ are characteristic of the $\mathrm{CH}_{3}$ protons, respectively.

Reaction of $\mathbf{2 a}-\mathbf{e}$ with acetic anhydride produced the pyrazole acetate derivatives $\mathbf{3 a}-\mathbf{e}$ in good yields. The structures of $\mathbf{3 a}-\mathbf{e}$ was confirmed by their IR, ${ }^{1} \mathrm{H}-\mathrm{NMR}$ and ${ }^{13} \mathrm{C}-\mathrm{NMR}$ spectra. The IR spectra of 3a-e showed the characteristic bands for $\mathrm{C}=\mathrm{N}$ at $1632-1656 \mathrm{~cm}^{-1}$, ester carbonyl band at 1746-1752 $\mathrm{cm}^{-1}$ and the $\mathrm{NH}_{2}$ band appeared at 3379-3393 and 3250-3330 $\mathrm{cm}^{-1}$. Also, the ${ }^{1} \mathrm{H}-\mathrm{NMR}$ spectra of 3a-e revealed the following signals: a singlet equivalent to two protons at $\delta=9.33-10.37 \mathrm{ppm}$ characteristic of $\mathrm{NH}_{2}$ protons, a multiplet at $\delta=7.13-8.29 \mathrm{ppm}$ characteristic for the aromatic protons, a singlet for the pyrazole $\mathrm{C}_{4}-\mathrm{H}$ at $\delta=6.72-6.93 \mathrm{ppm}$, beside the presence of two singlets at $\delta=2.22-2.39 \mathrm{ppm}$ and $\delta=3.21-3.33 \mathrm{ppm}$ corresponding to the methyl protons of $\mathrm{OCOCH}_{3}$ and $\mathrm{OCH}_{3}$, respectively. Moreover, the ${ }^{13} \mathrm{C}-\mathrm{NMR}$ spectrum of $\mathbf{3 d}$, as an example of this series, showed different characteristic signals at $\delta 20.7\left(\mathrm{CH}_{3}\right), 56.0\left(\mathrm{OCH}_{3}\right), 168.8(\mathrm{C}=\mathrm{N})$ and $189.3(\mathrm{C}=\mathrm{O})$.

Condensation of chalcones $1 \mathbf{a}-\mathbf{e}$ with either thiosemicarbazide or isonicotinic acid hydrazide in ethanol containing a few drops of acetic acid afforded the 4,5-dihydropyrazole derivatives $\mathbf{4 a - e}$ and 5a-e, respectively. The key reactions involved the intermediate formation of the hydrazones and subsequent addition of N-H on the olefinic bond of the propenone moiety that forms the ring-closed final products. The spectral data (IR, ${ }^{1} \mathrm{H}-\mathrm{NMR}$ and $\left.{ }^{13} \mathrm{C}-\mathrm{NMR}\right)$ of $\mathbf{4 a}-\mathbf{e}$ and $\mathbf{5 a}-\mathbf{e}$ were in full agreement with the proposed structures. The absence of the carbonyl $(\mathrm{CO})$ and olefinic $(\mathrm{C}=\mathrm{C})$ bands in the IR spectra of the 4,5-dihydropyrazole derivatives $4 \mathbf{a}-\mathbf{e}$ and $\mathbf{5 a}-\mathbf{e}$ proved the ring closure of the final products. The IR spectra of $\mathbf{4 a}-\mathbf{e}$ showed thiocarbonyl $\mathrm{C}=\mathrm{S}$ stretching bands at $1232-1248 \mathrm{~cm}^{-1}$ and $\mathrm{NH}_{2}$ absorption bands at 3387-3394 $\mathrm{cm}^{-1}$, whereas the IR spectra of 5a-e showed an amide carbonyl stretching band at $1628-1634 \mathrm{~cm}^{-1}$. Also, the ${ }^{1} \mathrm{H}-\mathrm{NMR}$ spectra of either $4 \mathbf{a}-\mathbf{e}$ or $\mathbf{5 a}-\mathbf{e}$, revealed the presence of a pair of doublets of doublets corresponding to the ring protons ( $\mathrm{H}_{\mathrm{A}}$ and $\mathrm{H}_{\mathrm{B}}$ ) of 4,5-dihydropyrazole. The $\mathrm{CH}$ protons $(\mathrm{Hx})$ appeared as doublets of doublets due to vicinal coupling with the two magnetically non-equivalent protons of the methylene group $\mathrm{H}_{\mathrm{A}}$ (up field shift of $\mathrm{CH}_{2}$ ) and $\mathrm{HB}_{\mathrm{B}}$ (downfield shift of $\left.\mathrm{CH}_{2}\right)$ at position 4 of the dihydropyrazole ring $\left(J_{\mathrm{AB}}=16 \mathrm{~Hz}, J_{\mathrm{AX}}=3.6 \mathrm{~Hz}\right.$, $J_{\mathrm{BX}}=12 \mathrm{~Hz}$ ). Moreover, the $\mathrm{NH}_{2}$ protons of the thiocarbamoyl group of the dihydropyrazoles $4 \mathbf{a}-\mathbf{e}$ appeared at $\delta=10.71-10.93 \mathrm{ppm}$, generally as broad bands. At the same time the ${ }^{13} \mathrm{C}-\mathrm{NMR}$ spectrum of $\mathbf{4 b}$ showed different signals at $\delta: 48.2,70.6$ ( $\mathrm{C}_{3}$ and $\mathrm{C}_{4}$ dihydropyrazole), 56.2, $56.5\left(2 \mathrm{OCH}_{3}\right)$, $169.4(\mathrm{C}=\mathrm{N}), 177.8(\mathrm{C}=\mathrm{S})$ and that for $5 \mathrm{c}$ revealed the signals at $\delta: 40.3,66.8\left(\mathrm{C}_{3}\right.$ and $\mathrm{C}_{4}$ dihydropyrazole), $56.3\left(\mathrm{OCH}_{3}\right), 164.3(\mathrm{C}=\mathrm{N}), 188.4(\mathrm{C}=\mathrm{O})$. All the results are shown in Scheme 1 . 


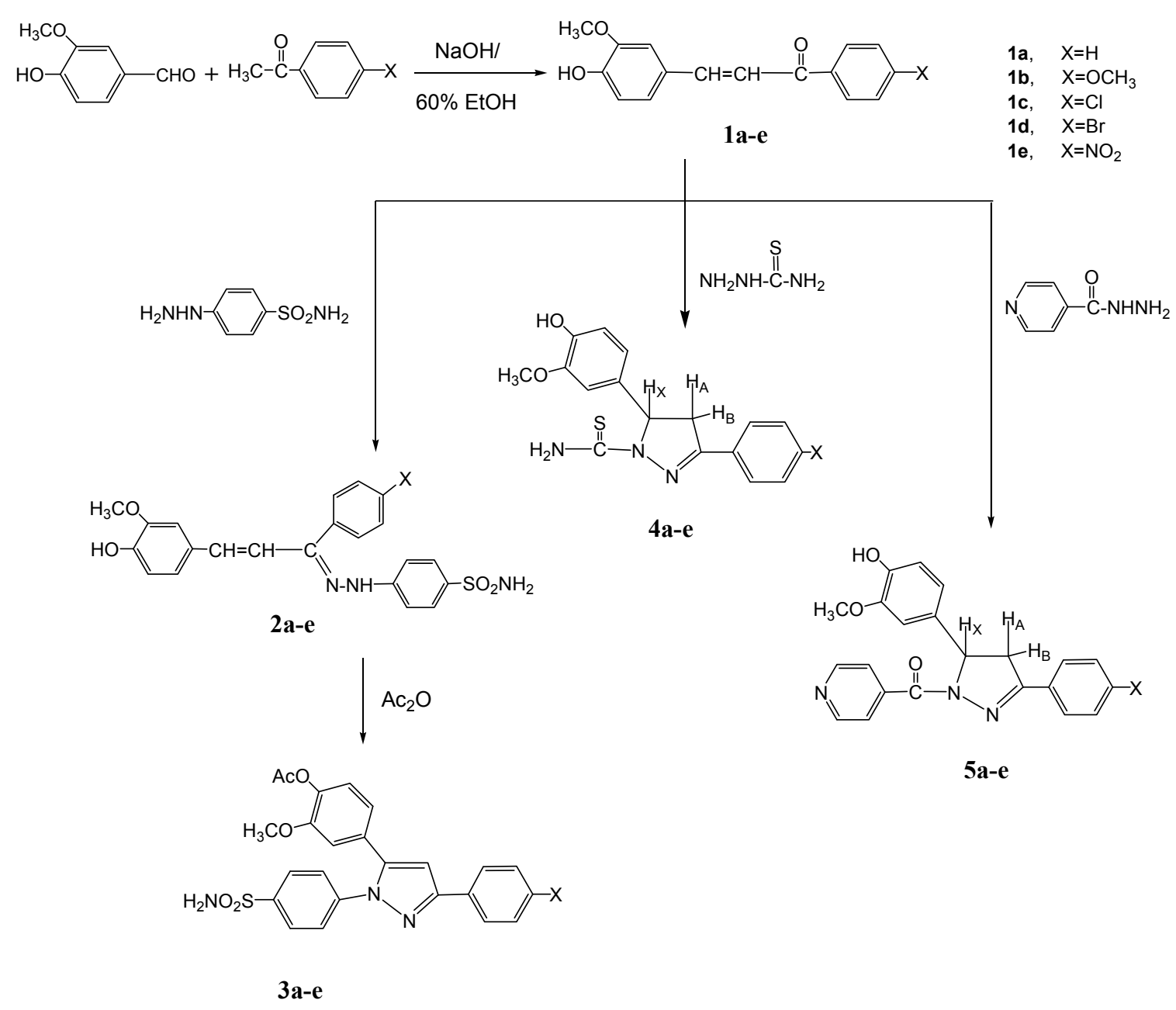

Scheme 1. Synthesis of some novel substituted pyrazoles.

\subsection{Pharmacological Activity}

\subsubsection{In Vitro Antibacterial Screening of Synthesized Compounds}

All of our synthesized compounds, chalcones $\mathbf{1 a}-\mathbf{e}$, hydrazones $\mathbf{2 a - e}$, pyrazoles $\mathbf{3 a}-\mathbf{e}$, and dihydropyrazoles $\mathbf{4 a - e}$ and $\mathbf{5 a}-\mathbf{e}$ were tested for their antimicrobial activity against four test organisms, namely Staphylococcus aureus ATCC6538P, Escherichia coli ATCC8739, Pseudomonas aeruginosa ATCC9027, and Candida albicans ATCC2091 using rifampicin (5 $\mu \mathrm{g} / \mathrm{disc})$ and ampicillin (10 $\mu \mathrm{g} / \mathrm{disc})$ as standard drugs. The agar well-diffusion method [46] was used for studying the potential activities of these compounds. Pyrazoles 3a-e showed no significant effect, whereas all other compounds showed potent activity only against Staphylococcus aureus and Candida albicans in the following order: $\mathbf{2 a - e}$ $>$ 4a-e $>$ 1a-e $\geq \mathbf{5 a}-\mathbf{e}$. Minimum inhibitory concentration (MIC) values for the individual compounds that showed inhibition zones $>10 \mathrm{~mm}$ were determined by means of the agar well-diffusion method in DMSO. The trend of activity was observed as follows: $\mathrm{X}>\mathrm{H}>\mathrm{OMe}>\mathrm{NO}_{2}$ where $\mathrm{X}=\mathrm{Cl}, \mathrm{Br}$. It is obvious that the presence of pharmacophores such as chloro and bromo substituents with lipophilic properties increases the antimicrobial activity. The activity results of our synthesized compounds against S. aureus, C. albicans are shown in Table 1 as zone of inhibition (in $\mathrm{mm}$ ) and minimum inhibitory concentration, MIC (mg/mL). 
Table 1. Determination of zone of inhibition and minimum inhibitory concentrations (MIC).

\begin{tabular}{|c|c|c|c|c|}
\hline \multirow{2}{*}{ Compound No. } & \multicolumn{2}{|c|}{ Zone of Inhibition (mm) } & \multicolumn{2}{|c|}{ Minimum Inhibitory Concentration (MIC) $\mathrm{mg} / \mathrm{mL}$} \\
\hline & S. aureus & C. albicans & S. aureus & C. albicans \\
\hline 1a & - & 15 & - & - \\
\hline $1 \mathrm{~b}$ & - & 15 & - & - \\
\hline $1 \mathrm{c}$ & 21 & 20 & 0.1 & 0.05 \\
\hline 1d & 15 & 18 & 0.063 & 0.063 \\
\hline 1e & - & 15 & - & - \\
\hline 2a & 19 & 22 & 0.063 & 0.031 \\
\hline $2 \mathbf{b}$ & 18 & 25 & 0.125 & 0.031 \\
\hline $2 c$ & 22 & 26 & 0.05 & 0.05 \\
\hline 2d & 18 & 20 & 0.063 & 0.125 \\
\hline $2 e$ & 17 & 17 & - & - \\
\hline $4 a$ & - & 15 & - & - \\
\hline $4 b$ & - & 15 & - & - \\
\hline $4 c$ & 21 & 24 & 0.05 & 0.05 \\
\hline $4 d$ & - & 15 & - & - \\
\hline $4 e$ & 17 & 17 & - & - \\
\hline $5 a$ & - & 20 & - & 0.25 \\
\hline $5 \mathbf{b}$ & - & 15 & - & - \\
\hline $5 c$ & 17 & 20 & 0.1 & 0.05 \\
\hline $5 d$ & 14 & 20 & 0.12 & 0.5 \\
\hline $5 e$ & 12 & 15 & - & - \\
\hline Rifampicin & 32 & - & - & - \\
\hline Ampicillin & 30 & - & - & - \\
\hline DMSO & - & 14 & - & - \\
\hline
\end{tabular}

Also, minimum bactericidal concentrations (MBC) were determined for all the chloro derivatives 1c, $2 \mathbf{c}, 4 \mathbf{c}$ and $\mathbf{5 c}$ which exhibited high activities. These results were listed in Table 2.

Table 2. Determination of minimum bactericidal concentration $(\mathrm{MBC})$ of the chloro series.

\begin{tabular}{c|ccccccc|cccccc}
\hline Concentrations mg/mL & $\mathbf{1}$ & $\mathbf{0 . 5 0}$ & $\mathbf{0 . 2 5}$ & $\mathbf{0 . 1 2 5}$ & $\mathbf{0 . 0 6 3}$ & $\mathbf{0 . 0 3 1}$ & $\mathbf{1}$ & $\mathbf{0 . 5 0}$ & $\mathbf{0 . 2 5}$ & $\mathbf{0 . 1 2 5}$ & $\mathbf{0 . 0 6 3}$ & $\mathbf{0 . 0 3 1}$ \\
\hline Microorganism Growth & \multicolumn{8}{|c}{ S. aureus } \\
\hline 1c & - & - & $*$ & + & + & + & - & $*$ & + & + & + & + \\
$\mathbf{2 c}$ & - & - & $*$ & + & + & + & - & - & $*$ & + & + & + \\
4c & - & $*$ & + & + & + & + & - & - & - & $*$ & + & + \\
5c & - & - & $*$ & + & + & + & - & - & - & $*$ & + & + \\
\hline
\end{tabular}

-: No turbidity; +: Turbidity; *: MBC

\subsubsection{Evaluation of Antioxidant and Anti-inflammatory Activities}

Two pharmacological activities, namely antioxidant and anti-inflammatory activities, were tested for the chloro derivatives $\mathbf{1 c}, \mathbf{2 c}, \mathbf{3 c}, \mathbf{4 c}, \mathbf{5 c}$. These activities vary according to their structures and functional groups. 


\section{Antioxidant Activity (DPPH Based Free Radical Scavenging Activity)}

The 1,1-diphenyl-2-picrylhydrazyl radical (DPPH) has been widely used to evaluate the free radical scavenging capacity of different antioxidants [47-50].

Resulting from a color change from purple to yellow, the absorbance decreased when the DPPH is scavenged by an antioxidant, through donation of hydrogen to form a stable DPPH molecule, in the radical form this molecule had an absorbance at $517 \mathrm{~nm}$, which disappeared after acceptance of an electron or hydrogen radical from an antioxidant compound to form the reduced DPPH-R (Scheme 2).

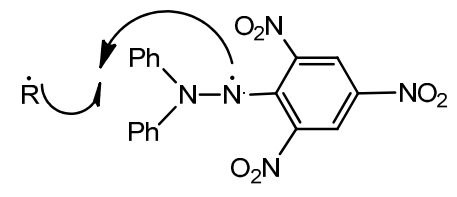

(Purple)

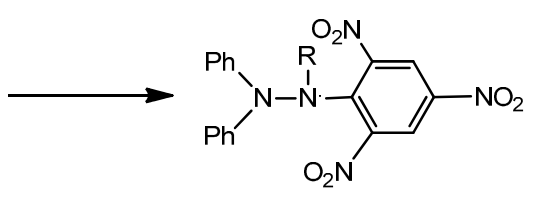

(Yellow)

Scheme 2. Reactions of DPPH.

Moreover antioxidants are known to interrupt the free-radical chain of oxidation and to donate hydrogen from phenolic hydroxy groups, thereby, forming stable free radicals, which do not initiate further oxidation [51]. Furthermore, substitution in the aromatic ring system with halogens like chlorine or bromine sharply enhanced the antioxidant potency [52], it is thought that the chlorine atom because of its lone pair electron as well as its electronegative power enhanced the formation and subsequent stabilization of the nitrogen-ring radical through intervening aromatic system property, it might have enhanced the power to absorb free radicals, especially reactive oxygen and reactive nitrogen species (ROS and RNS), this is explain why we select this series of our synthesized compounds. The present investigation emphasized mainly on the chloro derivatives which showed significant antioxidant activity, the screening of the selected synthesized compounds through structure-activity relationship (SAR) showed that compound $2 \mathrm{c}$ was found to be the most efficacious antioxidant among all the listed compounds. The antioxidant activity of $\mathbf{2 c}$ is directly proportional to the concentrations used. Antioxidant results of the synthesized compounds $\mathbf{1 c}, \mathbf{2 c}, \mathbf{3 c}, \mathbf{4 c}$ and $\mathbf{5 c}$ are reported in Table 3 . As reported in literature some substances can serve as either antioxidants or pro-oxidants, depending on conditions [53,54]. All the other tested compounds act as antioxidants at low concentrations $(0.25 \mathrm{mg} / \mathrm{mL})$ in the following order: $\mathbf{4 c}>\mathbf{5 c}>\mathbf{3 c}>\mathbf{1 c}$, while converted to pro-oxidant compounds at higher concentrations.

Anti-Inflammatory Activity (Scavenging of Nitric Oxide Radical)

Nitric oxide (NO) is a potent inhibitor of physiological processes such as smooth muscle relaxation, neuronal signaling, and inhibition of platelet aggregation and regulation of cell mediated toxicity [55]. In addition to reactive oxygen species, nitric oxide is also implicated in inflammation, cancer and other pathological conditions [56,57]. NO is known to be a ubiquitous free-radical moiety, which is distributed in tissues or organ systems and is supposed to have a vital role in neuromodulation or as a neurotransmitter in the CNS [58]. In our study all the chloro derivatives of the synthesized compounds $\mathbf{1 c}, \mathbf{2 c}, \mathbf{3 c}, \mathbf{4 c}, \mathbf{5 c}$ were tested for in vitro anti-inflammatory activity compared to the standard vitamin $\mathrm{C}$, showing 
acceptable anti-inflammatory activity. All tested compounds act as anti-inflammatory in a concentration dependent matter. Among all the tested compounds, $\mathbf{4 c}$ was the most potent compound, followed by: $\mathbf{5 c}>\mathbf{3 c}>\mathbf{1 c}>\mathbf{2 c}$. The in vitro anti-inflammatory activity of tested compounds is summarized in Table 4 .

Table 3. In vitro antioxidant activity data (DPPH scavenging).

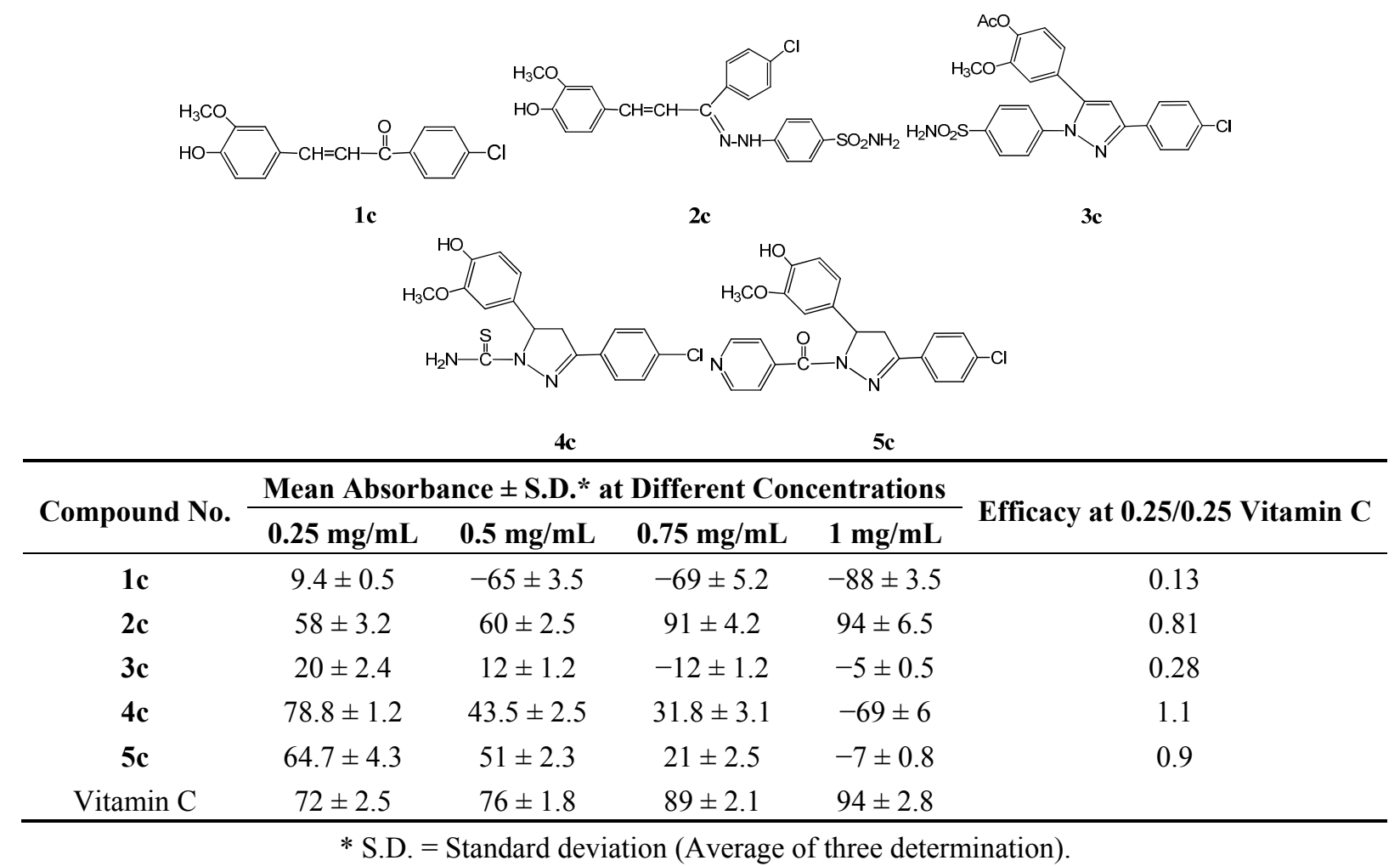

Table 4. In vitro anti-inflammatory activity data (NO scavenging).

\begin{tabular}{|c|c|c|c|c|c|}
\hline \multirow{2}{*}{ Compound No. } & \multicolumn{4}{|c|}{ Mean Absorbance \pm S.D.* at Different Concentrations } & \multirow{2}{*}{ Efficacy at $0.25 / 0.25$ Vitamin $C$} \\
\hline & $0.25 \mathrm{mg} / \mathrm{mL}$ & $0.5 \mathrm{mg} / \mathrm{mL}$ & $0.75 \mathrm{mg} / \mathrm{mL}$ & $1 \mathrm{mg} / \mathrm{mL}$ & \\
\hline $1 \mathrm{c}$ & $36 \pm 1.3$ & $38 \pm 2.1$ & $41 \pm 4.1$ & $44 \pm 1.5$ & 0.88 \\
\hline $2 c$ & $17 \pm 1.7$ & $19 \pm 1.2$ & $25 \pm 1.5$ & $29 \pm 1.8$ & 0.4 \\
\hline $3 c$ & $7 \pm 1.1$ & $14 \pm 1.2$ & $25 \pm 1.5$ & $47 \pm 3.5$ & 0.94 \\
\hline $4 c$ & $19 \pm 1.9$ & $36 \pm 3.2$ & $58 \pm 2.4$ & $74 \pm 4.5$ & 1.5 \\
\hline $5 c$ & $26 \pm 1.9$ & $34 \pm 1.5$ & $42 \pm 2.5$ & $62 \pm 5.2$ & 1.03 \\
\hline Vitamin C & & & & $50 \pm 1.2$ & \\
\hline
\end{tabular}

* S.D. $=$ Standard deviation (Average of three determination).

\section{Experimental Section}

\subsection{General Information}

Melting points were determined in open capillary tubes using Electrothermal apparatus 9100 (Fisher Scientific, Leicestershire, UK) and are uncorrected. Microanalyses were operated at Faculty of Science, Cairo University, Cairo, Egypt, using an Elementary Vario El III C, H, N, S Analyzer (Shimadzu, Berlin, Germany). IR spectra were recorded using the potassium bromide method on a Tensor 37 FT-IR spectrometer (Bruker, Ettlingen, Germany): and expressed in wave number $\left(v_{\max }\right) \mathrm{cm}^{-1}$. 
${ }^{1} \mathrm{H}-\mathrm{NMR}$ and ${ }^{13} \mathrm{C}-\mathrm{NMR}$ spectra were measured in deuterated chloroform $\left(\mathrm{CDCl}_{3}\right)$ or deuterated dimethyl sulphoxide (DMSO-d6) on an EAC 500 MHz FT-NMR spectrophotometer (Jeol, Eching, Germany). Chemical Shifts were recorded in $\delta$ as parts per million (ppm) downfield from tetramethylsilane (TMS) used as internal standard. Reaction progress and compound purity were monitored by Thin Layer Chromatography (TLC) using Alugram Sil G/UV254 silica gel plates (Macherey-Nagel, Easlon, PA, USA) and chloroform or chloroform-ethanol (9:1) or (19:1) as eluent systems. The spots were visualized using an ultraviolet lamp (Vilber Lourmet, Marine La Vallee, France) at $\lambda=254$ and $266 \mathrm{~nm}$. The antimicrobial activities were determined at the Department of Pharmaceutical Microbiology, Faculty of Pharmacy, Alexandria University. Antioxidant and anti-inflammatory activity tests were performed at the Biochemistry Lab, Faculty of Science, Alexandria University.

\subsubsection{General Procedure for the Preparation of $\mathbf{1 a}-\mathbf{e}$}

The appropriate $p$-substituted acetophenone $(10 \mathrm{mmol})$ was added to a cold stirred solution of sodium hydroxide ( $3 \mathrm{~g}$ in $50 \mathrm{~mL}$ of $60 \%$ aqueous ethanol) followed by addition of vanillin (10 mmol) dropwise with continuous stirring for five hours. The resulting crude solid was filtered, washed successively with water, dried and crystallized from ethanol (95\%) [44,45,59-61].

3-(4-Hydroxy-3-methoxyphenyl)-1-(phenyl)prop-2-en-1-one (1a). The chalcone was obtained in 73\% yield; m.p. $93{ }^{\circ} \mathrm{C}$; IR (KBr, cm $\left.{ }^{-1}\right)$ : $3426(\mathrm{OH}), 1666(\mathrm{C}=\mathrm{O}), \mathrm{CH}=\mathrm{CH}(1593) ;{ }^{1} \mathrm{H}-\mathrm{NMR}$ (DMSO-d6): $\delta 3.35\left(\mathrm{~s}, 3 \mathrm{H}, \mathrm{OCH}_{3}\right), 6.88(\mathrm{~d}, 1 \mathrm{H}, \mathrm{COCH}=\mathrm{CH}, J=15 \mathrm{~Hz}), 7.26(\mathrm{~d}, 1 \mathrm{H}, \mathrm{COCH}=\mathrm{CH}, J=15 \mathrm{~Hz})$, 7.34-7.86 (m, 8H, Ar-H), 11.32 (s, 1H, OH, $\mathrm{D}_{2} \mathrm{O}$ exchangeable); Anal. Calcd. for $\mathrm{C}_{16} \mathrm{H}_{14} \mathrm{O}_{3}$ : C, 75.57; H, 5.55. Found: C, 75.42; H, 5.97.

3-(4-Hydroxy-3-methoxyphenyl)-1-(4-methoxyphenyl) prop-2-en-1-one (1b). The chalcone was obtained in 68\% yield; m.p. $117{ }^{\circ} \mathrm{C}$; IR $\left(\mathrm{KBr}, \mathrm{cm}^{-1}\right): 3441(\mathrm{OH}), 1661(\mathrm{C}=\mathrm{O}), \mathrm{CH}=\mathrm{CH}(1502)$; ${ }^{1} \mathrm{H}-\mathrm{NMR}$ (DMSO-d $): \delta 3.21\left(\mathrm{~s}, 3 \mathrm{H}, \mathrm{OCH}_{3}\right), 3.33\left(\mathrm{~s}, 3 \mathrm{H}, \mathrm{OCH}_{3}\right), 6.72(\mathrm{~d}, 1 \mathrm{H}, \mathrm{COCH}=\mathrm{CH}, J=15 \mathrm{~Hz}), 7.25(\mathrm{~d}$, $1 \mathrm{H}, \mathrm{COCH}=\mathrm{CH}, J=15 \mathrm{~Hz}), 7.32-7.85(\mathrm{~m}, 7 \mathrm{H}, \mathrm{Ar}-\mathrm{H}), 11.44\left(\mathrm{~s}, 1 \mathrm{H}, \mathrm{OH}, \mathrm{D}_{2} \mathrm{O}\right.$ exchangeable); Anal. Calcd for $\mathrm{C}_{17} \mathrm{H}_{16} \mathrm{O}_{4}$ : C, 71.82; H, 5.67. Found: C, 72.00; H, 5.61.

1-(4-Chlorophenyl)-3-(4-hydroxy-3-methoxyphenyl)prop-2-en-1-one (1c). The chalcone was obtained in 77\% yield; m.p. $105{ }^{\circ} \mathrm{C}$; IR $\left(\mathrm{KBr}, \mathrm{cm}^{-1}\right): 3433(\mathrm{OH}), 1664(\mathrm{C}=\mathrm{O}), \mathrm{CH}=\mathrm{CH}(1614)$; ${ }^{1} \mathrm{H}-\mathrm{NMR}$ (DMSO-d6): $\delta 3.31\left(\mathrm{~s}, 3 \mathrm{H}, \mathrm{OCH}_{3}\right), 6.61(\mathrm{~d}, 1 \mathrm{H}, \mathrm{COCH}=\mathrm{CH}, J=15 \mathrm{~Hz}), 7.28(\mathrm{~d}, 1 \mathrm{H}, \mathrm{COCH}=\mathrm{CH}$, $J=15 \mathrm{~Hz}), 7.33-7.87(\mathrm{~m}, 7 \mathrm{H}, \mathrm{Ar}-\mathrm{H}), 11.44\left(\mathrm{~s}, 1 \mathrm{H}, \mathrm{OH}, \mathrm{D}_{2} \mathrm{O}\right.$ exchangeable $)$; Anal. Calcd for $\mathrm{C}_{16} \mathrm{H}_{13} \mathrm{ClO}_{3}$ : C, 66.56; H, 4.54. Found: C, 66.41; H, 4.57.

1-(4-Bromophenyl)-3-(4-hydroxy-3-methoxyphenyl) prop-2-en-1-one (1d). The chalcone was obtained in 82\% yield; m.p. $97{ }^{\circ} \mathrm{C}$; IR (KBr, cm ${ }^{-1}$ ): $3429(\mathrm{OH}), 1672(\mathrm{C}=\mathrm{O}), \mathrm{CH}=\mathrm{CH}(1606) ;{ }^{1} \mathrm{H}-\mathrm{NMR}$ (DMSO- $d 6$ ): $\delta 3.36\left(\mathrm{~s}, 3 \mathrm{H}, \mathrm{OCH}_{3}\right), 6.89(\mathrm{~d}, 1 \mathrm{H}, \mathrm{COCH}=\mathrm{CH}, J=15 \mathrm{~Hz}), 7.22(\mathrm{~d}, 1 \mathrm{H}, \mathrm{COCH}=\mathrm{CH}, J=15 \mathrm{~Hz})$, 7.30-7.74 (m, 7H, Ar-H), $11.22\left(\mathrm{~s}, 1 \mathrm{H}, \mathrm{OH}, \mathrm{D}_{2} \mathrm{O}\right.$ exchangeable); Anal. Calcd for $\mathrm{C}_{16} \mathrm{H}_{13} \mathrm{BrO}_{3}$ : C, 57.68; H, 3.93. Found: C, 57.72; H, 3.82.

3-(4-Hydroxy-3-methoxyphenyl)-1-(4-nitrophenyl) prop-2-en-1-one (1e). The chalcone was obtained in $85 \%$ yield; m.p. $95^{\circ} \mathrm{C}$; IR $\left(\mathrm{KBr}, \mathrm{cm}^{-1}\right)$ : $3424(\mathrm{OH}), 1684(\mathrm{C}=\mathrm{O}), \mathrm{CH}=\mathrm{CH}(1618) ;{ }^{1} \mathrm{H}-\mathrm{NMR}$ (DMSO- $d 6$ ): 
$\delta 3.30\left(\mathrm{~s}, 3 \mathrm{H}, \mathrm{OCH}_{3}\right), 6.89(\mathrm{~d}, 1 \mathrm{H}, \mathrm{COCH}=\mathrm{CH}, J=15 \mathrm{~Hz}), 7.29(\mathrm{~d}, 1 \mathrm{H}, \mathrm{COCH}=\mathrm{CH}, J=15 \mathrm{~Hz})$, 7.36-7.97 (m, 7H, Ar-H), $11.72\left(\mathrm{~s}, 1 \mathrm{H}, \mathrm{OH}, \mathrm{D}_{2} \mathrm{O}\right.$ exchangeable); Anal. Calcd. for $\mathrm{C}_{16} \mathrm{H}_{13} \mathrm{NO}_{5}$ : C, 64.21; H, 4.38; N, 4.68. Found: C, 64.19; H, 4.31; N, 4.69.

\subsubsection{General Procedure for the Preparation of $\mathbf{2 a}-\mathbf{e}$}

A solution of chalcone $1 \mathbf{a}-\mathbf{e}(10 \mathrm{mmol})$ in ethanol $(95 \%, 30 \mathrm{~mL})$ was refluxed with the appropriate amount of $p$-sulphamylphenylhydrazine $(10 \mathrm{mmol})$ in glacial acetic acid $(2 \mathrm{~mL})$ for six hours. The reaction mixture was poured into crushed ice and kept overnight at room temperature. The separated crude solid was filtered off, washed successively with water, dried and crystallized from ethanol (95\%) to give $\mathbf{2} \mathbf{a}-\mathbf{e}$ as needles.

4-(2-(3-(4-Hydroxy-3-methoxyphenyl)-1-phenylallylidene)hydrazinyl)benzenesulfonamide (2a). The hydrazone was obtained in 67\% yield; m.p. $147{ }^{\circ} \mathrm{C}$; IR $\left(\mathrm{KBr}, \mathrm{cm}^{-1}\right): 3430(\mathrm{OH}), 3391,3322\left(\mathrm{NH}_{2}\right.$, $\mathrm{NH}), 1632(\mathrm{C}=\mathrm{N}), 1603(\mathrm{CH}=\mathrm{CH}) ;{ }^{1} \mathrm{H}-\mathrm{NMR}\left(\mathrm{DMSO}-d_{6}\right): \delta 3.33\left(\mathrm{~s}, 3 \mathrm{H}, \mathrm{OCH}_{3}\right), 6.85(\mathrm{~d}, 1 \mathrm{H}$, $\mathrm{N}=\mathrm{C}-\mathrm{CH}=\mathrm{C} H, J=13 \mathrm{~Hz}), 7.54(\mathrm{~d}, 1 \mathrm{H}, \mathrm{N}=\mathrm{C}-\mathrm{CH}=\mathrm{CH}, J=13 \mathrm{~Hz}), 7.33-7.82(\mathrm{~m}, 12 \mathrm{H}, \mathrm{Ar}-\mathrm{H}), 8.21$ (s, $1 \mathrm{H}, \mathrm{NH}, \mathrm{D}_{2} \mathrm{O}$ exchangeable), 9.23 (s, 2H, $\mathrm{NH}_{2}, \mathrm{D}_{2} \mathrm{O}$ exchangeable), 11.22 (s,1H, OH, $\mathrm{D}_{2} \mathrm{O}$ exchangeable). Anal. Calcd. for $\mathrm{C}_{22} \mathrm{H}_{21} \mathrm{~N}_{3} \mathrm{O} 4 \mathrm{~S}$ : C, 62.40; H, 5.00; N, 9.92. Found: C, 62.47; H, 4.89; N, 9.88.

4-(2-(3-(4-Hydroxy-3-methoxyphenyl)-1-(4-methoxyphenylallylidene)hydrazinyl)benzenesulfonamide

(2b). The hydrazone was obtained in $90 \%$ yield; m.p. $160{ }^{\circ} \mathrm{C}$; IR $\left(\mathrm{KBr}, \mathrm{cm}^{-1}\right): 3432(\mathrm{OH}), 3389,3320$ $\left(\mathrm{NH}_{2}, \mathrm{NH}\right), 1629(\mathrm{C}=\mathrm{N}), 1609(\mathrm{CH}=\mathrm{CH}) ;{ }^{1} \mathrm{H}-\mathrm{NMR}\left(\mathrm{DMSO}-d_{6}\right): \delta 3.22\left(\mathrm{~s}, 3 \mathrm{H}, \mathrm{OCH}_{3}\right), 3.26(\mathrm{~s}, 3 \mathrm{H}$, $\left.\mathrm{OCH}_{3}\right), 6.83(\mathrm{~d}, 1 \mathrm{H}, \mathrm{N}=\mathrm{C}-\mathrm{CH}=\mathrm{C} H, J=13 \mathrm{~Hz}), 7.53(\mathrm{~d}, 1 \mathrm{H}, \mathrm{N}=\mathrm{C}-\mathrm{CH}=\mathrm{CH}, J=13 \mathrm{~Hz}), 7.31-7.81$ (m, $11 \mathrm{H}, \mathrm{Ar}-\mathrm{H}), 8.46$ (s, $1 \mathrm{H}, \mathrm{NH}, \mathrm{D}_{2} \mathrm{O}$ exchangeable), 9.29 (s, 2H, $\mathrm{NH}_{2}, \mathrm{D}_{2} \mathrm{O}$ exchangeable), 11.31 (s, $1 \mathrm{H}$, $\mathrm{OH}, \mathrm{D}_{2} \mathrm{O}$ exchangeable). Anal. Calcd. for $\mathrm{C}_{23} \mathrm{H}_{23} \mathrm{~N}_{3} \mathrm{O}_{5} \mathrm{~S}: \mathrm{C}, 60.91 ; \mathrm{H}, 5.11 ; \mathrm{N}, 9.27$. Found: C, 60.87; H, 5.08; N, 9.23.

4-(2-(1-(4-Chlorophenyl)-3-(4-hydroxy-3-methoxyphenylallylidene)hydrazinyl)benzenesulfonamide

(2c).

The hydrazone was obtained in $87 \%$ yield; m.p. $103{ }^{\circ} \mathrm{C}$; IR $\left(\mathrm{KBr}, \mathrm{cm}^{-1}\right)$ : $3439(\mathrm{OH}), 3382,3313\left(\mathrm{NH}_{2}\right.$, $\mathrm{NH}), 1626(\mathrm{C}=\mathrm{N}), 1612(\mathrm{CH}=\mathrm{CH}) ;{ }^{1} \mathrm{H}-\mathrm{NMR}\left(\mathrm{DMSO}-\mathrm{d}_{6}\right): \delta 3.22\left(\mathrm{~s}, 3 \mathrm{H}, \mathrm{OCH}_{3}\right), 6.69(\mathrm{~d}, 1 \mathrm{H}$, $\mathrm{N}=\mathrm{C}-\mathrm{CH}=\mathrm{C} H, J=13 \mathrm{~Hz}), 7.56(\mathrm{~d}, 1 \mathrm{H}, \mathrm{N}=\mathrm{C}-\mathrm{CH}=\mathrm{CH}, J=13 \mathrm{~Hz}), 7.36-7.61(\mathrm{~m}, 11 \mathrm{H}, \mathrm{Ar}-\mathrm{H}), 8.54(\mathrm{~s}$, $1 \mathrm{H}, \mathrm{NH}, \mathrm{D}_{2} \mathrm{O}$ exchangeable), $9.31\left(\mathrm{~s}, 2 \mathrm{H}, \mathrm{NH}_{2}, \mathrm{D}_{2} \mathrm{O}\right.$ exchangeable), $11.33\left(\mathrm{~s}, 1 \mathrm{H}, \mathrm{OH}, \mathrm{D}_{2} \mathrm{O}\right.$ exchangeable). Anal. Calcd. for $\mathrm{C}_{22} \mathrm{H}_{20} \mathrm{ClN}_{3} \mathrm{O} 4 \mathrm{~S}$ : C, 57.70; H, 4.40; N, 9.18. Found: C, 57.76; H, 4.35; N, 9.22.

4-(2-(1-(4-Bromophenyl)-3-(4-hydroxy-3-methoxyphenylallylidene)hydrazinyl)benzenesulfonamide (2d). The hydrazone was obtained in $93 \%$ yield; m.p. $110{ }^{\circ} \mathrm{C}$; IR $\left(\mathrm{KBr}, \mathrm{cm}^{-1}\right): 3435(\mathrm{OH}), 3383,3314\left(\mathrm{NH}_{2}\right.$, $\mathrm{NH}), 1633(\mathrm{C}=\mathrm{N}), 1604(\mathrm{CH}=\mathrm{CH}) ;{ }^{1} \mathrm{H}-\mathrm{NMR}\left(\mathrm{DMSO}-\mathrm{d}_{6}\right): \delta 3.19\left(\mathrm{~s}, 3 \mathrm{H}, \mathrm{OCH}_{3}\right), 6.81(\mathrm{~d}, 1 \mathrm{H}$, $\mathrm{N}=\mathrm{C}-\mathrm{CH}=\mathrm{C} H, J=13 \mathrm{~Hz}), 7.54(\mathrm{~d}, 1 \mathrm{H}, \mathrm{N}=\mathrm{C}-\mathrm{C} H=\mathrm{CH}, J=13 \mathrm{~Hz}), 7.41-7.71(\mathrm{~m}, 11 \mathrm{H}, \mathrm{Ar}-\mathrm{H}), 8.61$ (s, $1 \mathrm{H}, \mathrm{NH}, \mathrm{D}_{2} \mathrm{O}$ exchangeable), $9.39\left(\mathrm{~s}, 2 \mathrm{H}, \mathrm{NH}_{2}, \mathrm{D}_{2} \mathrm{O}\right.$ exchangeable), 11.39 (s, 1H, OH, $\mathrm{D}_{2} \mathrm{O}$ exchangeable). Anal. Calcd. for $\mathrm{C}_{22} \mathrm{H}_{20} \mathrm{BrN}_{3} \mathrm{O}_{4} \mathrm{~S}$ : C, 52.60; H, 4.01; N, 8.36. Found: C, 52.61; H, 3.89; $\mathrm{N}, 8.42$. 
4-(2-(3-(4-Hydroxy-3-methoxyphenyl)-1-(4-nitrophenylallylidene)hydrazinyl)benzenesulfonamide (2e). The hydrazone was obtained in $79 \%$ yield; m.p. $135{ }^{\circ} \mathrm{C}$; IR $\left(\mathrm{KBr}, \mathrm{cm}^{-1}\right): 3441(\mathrm{OH}), 3379,3310\left(\mathrm{NH}_{2}\right.$, $\mathrm{NH}), 1627(\mathrm{C}=\mathrm{N}), 1619(\mathrm{CH}=\mathrm{CH}) ;{ }^{1} \mathrm{H}-\mathrm{NMR}\left(\mathrm{DMSO}-\mathrm{d}_{6}\right): \delta 3.37\left(\mathrm{~s}, 3 \mathrm{H}, \mathrm{OCH}_{3}\right), 6.79(\mathrm{~d}, 1 \mathrm{H}$, $\mathrm{N}=\mathrm{C}-\mathrm{CH}=\mathrm{C} H, J=13 \mathrm{~Hz}), 7.61(\mathrm{~d}, 1 \mathrm{H}, \mathrm{N}=\mathrm{C}-\mathrm{C} H=\mathrm{CH}, J=13 \mathrm{~Hz}), 7.26-7.76(\mathrm{~m}, 11 \mathrm{H}, \mathrm{Ar}-\mathrm{H}), 8.73$ (s, 1H, NH, $\mathrm{D}_{2} \mathrm{O}$ exchangeable), 9.40 (s, 2H, $\mathrm{NH}_{2}, \mathrm{D}_{2} \mathrm{O}$ exchangeable), 11.38 (s, 1H, OH, $\mathrm{D}_{2} \mathrm{O}$ exchangeable). Anal. Calcd. for $\mathrm{C}_{22} \mathrm{H}_{20} \mathrm{~N}_{4} \mathrm{O}_{6} \mathrm{~S}$ : C, 56.40; H, 4.30; N, 11.96. Found: C, 56.39; H, 4.31; N, 11.95 .

\subsubsection{General Procedure for the Preparation of 3a-e}

A mixture of the appropriate hydrazone $\mathbf{2 a}-\mathbf{e}(10 \mathrm{mmol})$ and acetic anhydride $(15 \mathrm{~mL})$ was heated under reflux for three hours. After the reaction mixture attained room temperature, it was poured into crushed ice and the oily product deposited was decanted from water and extracted with ether. The ether layer was washed three times with water, dried over anhydrous sodium sulphate and evaporated. The precipitate obtained was crystallized from ethanol (95\%) to afford the corresponding pyrazoles $\mathbf{3 a}-\mathbf{e}$ as needles.

2-Methoxy-4-(3-phenyl-1-(4-sulfamoylphenyl)-1H-pyrazol-5-yl)phenyl acetate (3a). The pyrazole was obtained in 69\% yield; m.p. $187^{\circ} \mathrm{C}$; IR $\left(\mathrm{KBr}, \mathrm{cm}^{-1}\right)$ : $3386\left(\mathrm{NH}_{2}\right), 1750(\mathrm{C}=\mathrm{O}), 1632(\mathrm{C}=\mathrm{N})$; ${ }^{1} \mathrm{H}-\mathrm{NMR}$ (DMSO-d6): $\delta 2.39\left(\mathrm{~s}, 3 \mathrm{H}, \mathrm{CH}_{3}\right), 3.33\left(\mathrm{~s}, 3 \mathrm{H}, \mathrm{OCH}_{3}\right), 6.72\left(\mathrm{~s}, 1 \mathrm{H}\right.$, pyrazole $\left.\mathrm{C}_{4}-\mathrm{H}\right), 7.24-8.29(\mathrm{~m}, 12 \mathrm{H}$, Ar-H), 9.33 (s, 2H, NH $2, \mathrm{D}_{2} \mathrm{O}$ exchangeable). Anal. Calcd. for $\mathrm{C}_{24} \mathrm{H}_{21} \mathrm{~N}_{3} \mathrm{O} \mathrm{S}_{5} \mathrm{~S}: \mathrm{C}, 62.19 ; \mathrm{H}, 4.57$; N, 9.07. Found: C, 62.19; H, 4.40; N, 9.09.

2-Methoxy-4-(3-(4-methoxyphenyl)-1-(4-sulfamoylphenyl)-1H-pyrazol-5-yl)phenyl acetate (3b). The pyrazole was obtained in 91\% yield; m.p. $193{ }^{\circ} \mathrm{C}$; IR $\left(\mathrm{KBr}, \mathrm{cm}^{-1}\right): 3384\left(\mathrm{NH}_{2}\right), 1751(\mathrm{C}=\mathrm{O}), 1641$ $(\mathrm{C}=\mathrm{N}) ;{ }^{1} \mathrm{H}-\mathrm{NMR}\left(\mathrm{DMSO}-d_{6}\right): \delta 2.28\left(\mathrm{~s}, 3 \mathrm{H}, \mathrm{CH}_{3}\right), 3.21\left(\mathrm{~s}, 3 \mathrm{H}, \mathrm{OCH}_{3}\right), 3.34\left(\mathrm{~s}, 3 \mathrm{H}, \mathrm{OCH}_{3}\right), 6.87(\mathrm{~s}$, $1 \mathrm{H}$, pyrazole $\left.\mathrm{C}_{4}-\mathrm{H}\right), 7.35-7.94(\mathrm{~m}, 11 \mathrm{H}, \mathrm{Ar}-\mathrm{H}), 9.76\left(\mathrm{~s}, 2 \mathrm{H}, \mathrm{NH}_{2}, \mathrm{D}_{2} \mathrm{O}\right.$ exchangeable). Anal. Calcd. for $\mathrm{C}_{25} \mathrm{H}_{23} \mathrm{~N}_{3} \mathrm{O}_{6} \mathrm{~S}$ : C, 60.84; H, 4.70; N, 8.51. Found: C, 60.75; H, 4. 68; N, 8.59.

4-(3-(4-Chlorophenyl)-1-(4-sulfamoylphenyl)-1H-pyrazol-5-yl)-2-methoxyphenyl acetate (3c). The pyrazole was obtained in $76 \%$ yield; m.p. $172{ }^{\circ} \mathrm{C}$; IR $\left(\mathrm{KBr}, \mathrm{cm}^{-1}\right): 3379\left(\mathrm{NH}_{2}\right), 1748(\mathrm{C}=\mathrm{O}), 1644(\mathrm{C}=\mathrm{N})$; ${ }^{1} \mathrm{H}-\mathrm{NMR}$ (DMSO-d6): $\delta 2.22\left(\mathrm{~s}, 3 \mathrm{H}, \mathrm{CH}_{3}\right), 3.27\left(\mathrm{~s}, 3 \mathrm{H}, \mathrm{OCH}_{3}\right), 6.83\left(\mathrm{~s}, 1 \mathrm{H}\right.$, pyrazole $\left.\mathrm{C}_{4}-\mathrm{H}\right), 7.29-7.86$ (m, 11H, Ar-H), 9.92 (s, 2H, $\mathrm{NH}_{2}, \mathrm{D}_{2} \mathrm{O}$ exchangeable). Anal. Calcd. for $\mathrm{C}_{24} \mathrm{H}_{20} \mathrm{ClN}_{3} \mathrm{O}_{5} \mathrm{~S}: \mathrm{C}, 57.89 ; \mathrm{H}$, 4.05; N, 8.44. Found: C, 57.80; H, 4.10; N, 8.40.

4-(3-(4-Bromophenyl)-1-(4-sulfamoylphenyl)-1H-pyrazol-5-yl)-2-methoxyphenyl acetate (3d). The pyrazole was obtained in $78 \%$ yield; m.p. $177{ }^{\circ} \mathrm{C}$; IR $\left(\mathrm{KBr}, \mathrm{cm}^{-1}\right): 3391\left(\mathrm{NH}_{2}\right), 1746(\mathrm{C}=\mathrm{O}), 1654$ $(\mathrm{C}=\mathrm{N}) ;{ }^{1} \mathrm{H}-\mathrm{NMR}\left(\mathrm{CDCl}_{3}\right): \delta 2.30\left(\mathrm{~s}, 3 \mathrm{H}, \mathrm{CH}_{3}\right), 3.31\left(\mathrm{~s}, 3 \mathrm{H}, \mathrm{OCH}_{3}\right), 6.85\left(\mathrm{~s}, 1 \mathrm{H}\right.$, pyrazole $\left.\mathrm{C}_{4}-\mathrm{H}\right)$, 7.13-7.64 (m, 11H, Ar-H), $9.71\left(\mathrm{~s}, 2 \mathrm{H}, \mathrm{NH}_{2}, \mathrm{D}_{2} \mathrm{O}\right.$ exchangeable); ${ }^{13} \mathrm{C}-\mathrm{NMR}\left(\mathrm{CDCl}_{3}\right): \delta 20.7\left(\mathrm{CH}_{3}\right)$, $56.0\left(\mathrm{OCH}_{3}\right), 111.9,121.5,121.8,123.4,128.0,130.1,132.0,133.7,136.9,140.4,141.8,142.2,144.8$, 151.5 (pyrazole, $\mathrm{C}_{6} \mathrm{H}_{3}$ and $\left.2 \mathrm{C}_{6} \mathrm{H}_{4} \mathrm{C}\right), 168.8(\mathrm{C}=\mathrm{N})$ and $189.3(\mathrm{C}=\mathrm{O})$. Anal. Calcd. for $\mathrm{C}_{24} \mathrm{H}_{20} \mathrm{BrN}_{3} \mathrm{O} 5 \mathrm{~S}$ : C, 53.14; H, 3.72; N, 7.75. Found: C, 53.10; H, 3.66; N, 7.80. 
2-Methoxy-4-(3-(4-nitrophenyl)-1-(4-sulfamoylphenyl)-1H-pyrazol-5-yl)phenyl acetate (3e). The pyrazole was obtained in 69\% yield; m.p. $197{ }^{\circ} \mathrm{C}$; IR $\left(\mathrm{KBr}, \mathrm{cm}^{-1}\right): 3393\left(\mathrm{NH}_{2}\right), 1752(\mathrm{C}=\mathrm{O}), 1656(\mathrm{C}=\mathrm{N})$; ${ }^{1} \mathrm{H}-\mathrm{NMR}\left(\mathrm{DMSO}-d_{6}\right): \delta 2.26\left(\mathrm{~s}, 3 \mathrm{H}, \mathrm{CH}_{3}\right), 3.33\left(\mathrm{~s}, 3 \mathrm{H}, \mathrm{OCH}_{3}\right), 6.93\left(\mathrm{~s}, 1 \mathrm{H}\right.$, pyrazole $\left.\mathrm{C}_{4}-\mathrm{H}\right), 7.29-8.10$ (m, $11 \mathrm{H}, \mathrm{Ar}-\mathrm{H}), 10.37$ (s, 2H, $\mathrm{NH}_{2}, \mathrm{D}_{2} \mathrm{O}$ exchangeable). Anal. Calcd. for $\mathrm{C}_{24} \mathrm{H}_{20} \mathrm{~N}_{4} \mathrm{O} 7 \mathrm{~S}: \mathrm{C}, 56.69$; $\mathrm{H}, 3.96$; N, 11.02. Found: C, 56.72; H, 3.95; N, 11.10 .

\subsubsection{General Procedure for the Preparation of $\mathbf{4 a}-\mathbf{e}$}

A mixture of the appropriate chalcone $1 \mathbf{a}-\mathbf{e}(10 \mathrm{mmol})$ in ethanol $(30 \mathrm{~mL})$ was heated under reflux with thiosemicarbazide $(12 \mathrm{mmol})$ in glacial acetic acid $(2 \mathrm{~mL})$ for $7 \mathrm{~h}$, then the reaction mixture was poured into crushed ice and kept overnight at room temperature. The separated crude solid was filtered off, washed successively with water, dried and crystallized from ethanol/chloroform to give $\mathbf{4 a}-\mathbf{e}$ as needles.

5-(4-Hydroxy-3-methoxyphenyl)-3-phenyl-4,5-dihydro-1H-pyrazole-1-carbothioamide (4a). The 4,5-dihydropyrazole was obtained in $81 \%$ yield; m.p. $149^{\circ} \mathrm{C}$; IR $\left(\mathrm{KBr}, \mathrm{cm}^{-1}\right): 3430(\mathrm{OH}), 3394\left(\mathrm{NH}_{2}\right)$, $1647(\mathrm{C}=\mathrm{N}), 1246(\mathrm{C}=\mathrm{S}) ;{ }^{1} \mathrm{H}-\mathrm{NMR}\left(\mathrm{DMSO}-d_{6}\right): \delta 3.11\left(\mathrm{dd}, 1 \mathrm{H}\right.$, pyrazolyl-H $\mathrm{H}_{\mathrm{A}}, J_{\mathrm{AX}}=3.6 \mathrm{~Hz}$, $\left.J_{\mathrm{AB}}=16 \mathrm{~Hz}\right), 3.31\left(\mathrm{~s}, 3 \mathrm{H}, \mathrm{OCH}_{3}\right), 3.72\left(\mathrm{dd}, 1 \mathrm{H}\right.$, pyrazolyl-H $\left.\mathrm{B}, J_{\mathrm{BX}}=12 \mathrm{~Hz}, J_{\mathrm{AB}}=16 \mathrm{~Hz}\right), 5.39(\mathrm{dd}$, $1 \mathrm{H}$, pyrazolyl-Hx, $\left.J_{\mathrm{AX}}=3.6 \mathrm{~Hz}, J_{\mathrm{BX}}=12 \mathrm{~Hz}\right), 7.26-7.82(\mathrm{~m}, 8 \mathrm{H}, \mathrm{Ar}-\mathrm{H}), 10.73\left(\mathrm{~s}, 2 \mathrm{H}, \mathrm{NH}_{2}, \mathrm{D}_{2} \mathrm{O}\right.$ exchangeable), 11.21 (s, $1 \mathrm{H}, \mathrm{OH}, \mathrm{D}_{2} \mathrm{O}$ exchangeable). Anal. Calcd. for $\mathrm{C}_{17} \mathrm{H}_{17} \mathrm{~N}_{3} \mathrm{O}_{2} \mathrm{~S}$ : C, 62.36; H, 5.23; N, 12.83. Found: C, 62.33; H, 5.20; N, 12.88 .

5-(4-Hydroxy-3-methoxyphenyl)-3-(4-methoxyphenyl)-4,5-dihydro-1H-pyrazole-1-carbothioamide (4b). The 4,5-dihydropyrazole was obtained in $83 \%$ yield; m.p. $156{ }^{\circ} \mathrm{C}$; IR $\left(\mathrm{KBr}, \mathrm{cm}^{-1}\right): 3432(\mathrm{OH}), 3390$ $\left(\mathrm{NH}_{2}\right), 1650(\mathrm{C}=\mathrm{N}), 1245(\mathrm{C}=\mathrm{S})$; ${ }^{1} \mathrm{H}-\mathrm{NMR}\left(\mathrm{DMSO}-d_{6}\right): \delta 3.20\left(\mathrm{dd}, 1 \mathrm{H}\right.$, pyrazolyl-H $\mathrm{H}_{\mathrm{A}}, J_{\mathrm{AX}}=3.6 \mathrm{~Hz}$, $\left.J_{\mathrm{AB}}=16 \mathrm{~Hz}\right), 3.32\left(\mathrm{~s}, 3 \mathrm{H}, \mathrm{OCH}_{3}\right), 3.39\left(\mathrm{~s}, 3 \mathrm{H}, \mathrm{OCH}_{3}\right), 3.75\left(\mathrm{dd}, 1 \mathrm{H}\right.$, pyrazolyl-HB,$J_{\mathrm{BX}}=12 \mathrm{~Hz}$, $\left.J_{\mathrm{AB}}=16 \mathrm{~Hz}\right), 5.39\left(\mathrm{dd}, 1 \mathrm{H}\right.$, pyrazolyl-Hx, $\left.J_{\mathrm{AX}}=3.6 \mathrm{~Hz}, J_{\mathrm{BX}}=12 \mathrm{~Hz}\right), 7.27-7.77(\mathrm{~m}, 7 \mathrm{H}, \mathrm{Ar}-\mathrm{H}), 10.73$ (s, 2H, $\mathrm{NH}_{2}, \mathrm{D}_{2} \mathrm{O}$ exchangeable), $11.30\left(\mathrm{~s}, 1 \mathrm{H}, \mathrm{OH}, \mathrm{D}_{2} \mathrm{O}\right.$ exchangeable); ${ }^{13} \mathrm{C}-\mathrm{NMR}$ (DMSO- $d_{6}$ ): $\delta$ 48.2, $70.6\left(\mathrm{C}_{3}\right.$ and $\mathrm{C}_{4}$ dihydropyrazole $), 56.2,56.5\left(2 \mathrm{OCH}_{3}\right), 109.7,113.2,115.7,119.4,122.9$, 126.0, 134.3, 143.4, 148.6, $149.2\left(\mathrm{C}_{6} \mathrm{H}_{3}\right.$ and $\left.\mathrm{C}_{6} \mathrm{H}_{4} \mathrm{C}\right), 169.4(\mathrm{C}=\mathrm{N})$ and $177.8(\mathrm{C}=\mathrm{S})$. Anal. Calcd. for $\mathrm{C}_{18} \mathrm{H}_{19} \mathrm{~N}_{3} \mathrm{O}_{3} \mathrm{~S}$ : C, 60.49; H, 5.36; N, 11.76. Found: C, 60.50; H, 5.34; N, 11.81.

3-(4-Chlorophenyl)-5-(4-hydroxy-3-methoxyphenyl)-4,5-dihydro-1H-pyrazole-1-carbothioamide (4c). The 4,5-dihydropyrazole was obtained in $87 \%$ yield; m.p. $172{ }^{\circ} \mathrm{C}$; IR $\left(\mathrm{KBr}, \mathrm{cm}^{-1}\right): 3439(\mathrm{OH}), 3390$ $\left(\mathrm{NH}_{2}\right), 1652(\mathrm{C}=\mathrm{N}), 1248(\mathrm{C}=\mathrm{S}) ;{ }^{1} \mathrm{H}-\mathrm{NMR}\left(\mathrm{DMSO}-d_{6}\right): \delta 3.08\left(\mathrm{dd}, 1 \mathrm{H}\right.$, pyrazolyl-H $\mathrm{H}_{\mathrm{A}}, J_{\mathrm{AX}}=3.6 \mathrm{~Hz}$, $\left.J_{\mathrm{AB}}=16 \mathrm{~Hz}\right), 3.27\left(\mathrm{~s}, 3 \mathrm{H}, \mathrm{OCH}_{3}\right), 3.79\left(\mathrm{dd}, 1 \mathrm{H}\right.$, pyrazolyl-H $\left., J_{\mathrm{BX}}=12 \mathrm{~Hz}, J_{\mathrm{AB}}=16 \mathrm{~Hz}\right), 5.44(\mathrm{dd}$, $1 \mathrm{H}$, pyrazolyl-Hx, $\left.J_{\mathrm{Ax}}=3.6 \mathrm{~Hz}, J_{\mathrm{BX}}=12 \mathrm{~Hz}\right), 7.29-8.01(\mathrm{~m}, 7 \mathrm{H}, \mathrm{Ar}-\mathrm{H}), 10.79\left(\mathrm{~s}, 2 \mathrm{H}, \mathrm{NH}_{2}, \mathrm{D}_{2} \mathrm{O}\right.$ exchangeable), 11.33 (s, 1H, OH, $\mathrm{D}_{2} \mathrm{O}$ exchangeable). Anal. Calcd. for $\mathrm{C}_{17} \mathrm{H}_{16} \mathrm{ClN}_{3} \mathrm{O}_{2} \mathrm{~S}: \mathrm{C}, 56.43$; H, 4.46; N, 11.61. Found: C, 56.33; H, 4.42; N, 11.71 .

3-(4-Bromophenyl)-5-(4-hydroxy-3-methoxyphenyl)-4,5-dihydro-1H-pyrazole-1-carbothioamide (4d). The 4,5-dihydropyrazole was obtained in 79\% yield; m.p. $146{ }^{\circ} \mathrm{C}$; IR $\left(\mathrm{KBr}, \mathrm{cm}^{-1}\right): 3440(\mathrm{OH}), 3393$ $\left(\mathrm{NH}_{2}\right), 1657(\mathrm{C}=\mathrm{N}), 1247(\mathrm{C}=\mathrm{S})$; ${ }^{1} \mathrm{H}-\mathrm{NMR}\left(\mathrm{DMSO}-d_{6}\right): \delta 3.12\left(\mathrm{dd}, 1 \mathrm{H}\right.$, pyrazolyl-H $\mathrm{A}_{\mathrm{A}}, J_{\mathrm{AX}}=3.6 \mathrm{~Hz}$, 
$\left.J_{\mathrm{AB}}=16 \mathrm{~Hz}\right), 3.29\left(\mathrm{~s}, 3 \mathrm{H}, \mathrm{OCH}_{3}\right), 3.76\left(\mathrm{dd}, 1 \mathrm{H}\right.$, pyrazolyl-HB $\left., J_{\mathrm{BX}}=12 \mathrm{~Hz}, J_{\mathrm{AB}}=16 \mathrm{~Hz}\right), 5.46(\mathrm{dd}$, $1 \mathrm{H}$, pyrazolyl-Hx, $\left.J_{\mathrm{AX}}=3.6 \mathrm{~Hz}, J_{\mathrm{BX}}=12 \mathrm{~Hz}\right), 7.23-7.61(\mathrm{~m}, 7 \mathrm{H}, \mathrm{Ar}-\mathrm{H}), 10.93\left(\mathrm{~s}, 2 \mathrm{H}, \mathrm{NH}_{2}, \mathrm{D}_{2} \mathrm{O}\right.$ exchangeable), 11.27 (s, 1H, OH, $\mathrm{D}_{2} \mathrm{O}$ exchangeable). Anal. Calcd. for $\mathrm{C}_{17} \mathrm{H}_{16} \mathrm{BrN}_{3} \mathrm{O}_{2} \mathrm{~S}: \mathrm{C}, 50.25$; H, 3.97; N, 10.34. Found: C, 50.23; H, 3.89; N, 10.37.

5-(4-Hydroxy-3-methoxyphenyl)-3-(4-nitrophenyl)-4,5-dihydro-1H-pyrazole-1-carbothioamide

(4e).

The 4,5-dihydropyrazole was obtained in 93\% yield; m.p.: $166^{\circ} \mathrm{C}$; IR $\left(\mathrm{KBr}, \mathrm{cm}^{-1}\right): 3451(\mathrm{OH}), 3387$ $\left(\mathrm{NH}_{2}\right), 1646(\mathrm{C}=\mathrm{N}), 1232(\mathrm{C}=\mathrm{S}) ;{ }^{1} \mathrm{H}-\mathrm{NMR}\left(\mathrm{DMSO}-d_{6}\right): \delta 3.07\left(\mathrm{dd}, 1 \mathrm{H}\right.$, pyrazolyl-HA, $J_{\mathrm{AX}}=3.6 \mathrm{~Hz}$, $\left.J_{\mathrm{AB}}=16 \mathrm{~Hz}\right), 3.32\left(\mathrm{~s}, 3 \mathrm{H}, \mathrm{OCH}_{3}\right), 3.74\left(\mathrm{dd}, 1 \mathrm{H}\right.$, pyrazolyl-H $\left.\mathrm{B}, J_{\mathrm{BX}}=12 \mathrm{~Hz}, J_{\mathrm{AB}}=16 \mathrm{~Hz}\right), 3.39(\mathrm{dd}$, $1 \mathrm{H}$, pyrazolyl-Hx, $\left.J_{\mathrm{AX}}=3.6 \mathrm{~Hz}, J_{\mathrm{BX}}=12 \mathrm{~Hz}\right), 7.27-7.99(\mathrm{~m}, 7 \mathrm{H}, \mathrm{Ar}-\mathrm{H}), 10.71\left(\mathrm{~s}, 2 \mathrm{H}, \mathrm{NH}_{2}, \mathrm{D}_{2} \mathrm{O}\right.$ exchangeable), 11.24 (s, $1 \mathrm{H}, \mathrm{OH}, \mathrm{D}_{2} \mathrm{O}$ exchangeable). Anal. Calcd. for $\mathrm{C}_{17} \mathrm{H}_{16} \mathrm{~N}_{4} \mathrm{O} 4 \mathrm{~S}: \mathrm{C}, 54.83 ; \mathrm{H}, 4.33$; N, 15.04. Found: C, 54.87; H, 4.33; N, 15.08 .

\subsubsection{General Procedure for the Preparation of 5a-e}

A mixture of the appropriate chalcone $1 \mathbf{a}-\mathbf{e}(10 \mathrm{mmol})$ in ethanol $(95 \%)(30 \mathrm{~mL})$ was heated under reflux with isonicotinic acid hydrazide $(10 \mathrm{mmol})$ in glacial acetic acid $(2 \mathrm{~mL})$ for five hours. The reaction mixture was treated as mentioned for the preparation of $\mathbf{4 a}-\mathbf{e}$ to give the corresponding 4,5-dihydropyrazoles 5a-e.

(5-(4-Hydroxy-3-methoxyphenyl)-3-phenyl-4,5-dihydro-1H-pyrazol-1-yl)(pyridin-4-yl)methanone (5a). The corresponding 4,5-dihydropyrazole was obtained in $81 \%$ yield; m.p. $159{ }^{\circ} \mathrm{C}$; IR $\left(\mathrm{KBr}, \mathrm{cm}^{-1}\right): 3472$ $(\mathrm{OH}), 1645(\mathrm{C}=\mathrm{N}), 1630(\mathrm{C}=\mathrm{O}) ;{ }^{1} \mathrm{H}-\mathrm{NMR}\left(\mathrm{DMSO}-d_{6}\right): \delta 3.14\left(\mathrm{dd}, 1 \mathrm{H}\right.$, pyrazolyl-H $\mathrm{H}_{\mathrm{A}}, J_{\mathrm{AX}}=3.6 \mathrm{~Hz}$, $\left.J_{\mathrm{AB}}=16 \mathrm{~Hz}\right), 3.32\left(\mathrm{~s}, 3 \mathrm{H}, \mathrm{OCH}_{3}\right), 3.84\left(\mathrm{dd}, 1 \mathrm{H}\right.$, pyrazolyl-HB $\left., J_{\mathrm{BX}}=12 \mathrm{~Hz}, J_{\mathrm{AB}}=16 \mathrm{~Hz}\right), 5.40(\mathrm{dd}$, $1 \mathrm{H}$, pyrazolyl-Hx, $\left.J_{\mathrm{AX}}=3.6 \mathrm{~Hz}, J_{\mathrm{BX}}=12 \mathrm{~Hz}\right), 6.81-7.48(\mathrm{~m}, 12 \mathrm{H}, \mathrm{Ar}-\mathrm{H}), 11.27\left(\mathrm{~s}, 1 \mathrm{H}, \mathrm{OH}, \mathrm{D}_{2} \mathrm{O}\right.$ exchangeable). Anal. Calcd. for $\mathrm{C}_{22} \mathrm{H}_{19} \mathrm{~N}_{3} \mathrm{O}_{3}$ : C, 70.76; H, 5.13; N, 11.25. Found: C, 70.77; H, 5.12; $\mathrm{N}, 11.27$.

(5-(4-Hydroxy-3-methoxyphenyl)-3-(4-methoxyphenyl)-4,5-dihydro-1H-pyrazol-1-yl)(pyridin-4-yl) methanone (5b). The corresponding 4,5-dihydropyrazole was obtained in $69 \%$ yield; m.p. $166{ }^{\circ} \mathrm{C}$; IR $\left(\mathrm{KBr}, \mathrm{cm}^{-1}\right): 3492(\mathrm{OH}), 1649(\mathrm{C}=\mathrm{N}), 1628(\mathrm{C}=\mathrm{O})$; ${ }^{1} \mathrm{H}-\mathrm{NMR}(\mathrm{DMSO}-d 6): \delta 3.16(\mathrm{dd}, 1 \mathrm{H}$, pyrazolyl-HA, $\left.J_{\mathrm{AX}}=3.6 \mathrm{~Hz}, J_{\mathrm{AB}}=16 \mathrm{~Hz}\right), 3.25\left(\mathrm{~s}, 3 \mathrm{H}, \mathrm{OCH}_{3}\right), 3.31\left(\mathrm{~s}, 3 \mathrm{H}, \mathrm{OCH}_{3}\right), 3.75(\mathrm{dd}, 1 \mathrm{H}$, pyrazolyl-HB, $\left.J_{\mathrm{BX}}=12 \mathrm{~Hz}, J_{\mathrm{AB}}=16 \mathrm{~Hz}\right), 5.37\left(\mathrm{dd}, 1 \mathrm{H}\right.$, pyrazolyl-Hx, $\left.J_{\mathrm{AX}}=3.6 \mathrm{~Hz}, J_{\mathrm{BX}}=12 \mathrm{~Hz}\right), 6.79-7.49(\mathrm{~m}$, $11 \mathrm{H}, \mathrm{Ar}-\mathrm{H}), 11.31$ (s, $1 \mathrm{H}, \mathrm{OH}, \mathrm{D}_{2} \mathrm{O}$ exchangeable). Anal. Calcd. for $\mathrm{C}_{23} \mathrm{H}_{21} \mathrm{~N}_{3} \mathrm{O}_{4}$ : C, 68.47; H, 5.25; N, 10.42. Found: C, 68.51; H, 5.26; N, 10.48.

(3-(4-Chlorophenyl)-5-(4-hydroxy-3-methoxyphenyl)-4,5-dihydro-1H-pyrazol-1-yl)(pyridin-4-yl) methanone (5c). The corresponding 4,5-dihydropyrazole was obtained in $89 \%$ yield; m.p. $142{ }^{\circ} \mathrm{C}$; IR $\left(\mathrm{KBr}, \mathrm{cm}^{-1}\right): 3481(\mathrm{OH}), 1655(\mathrm{C}=\mathrm{N}), 1631(\mathrm{C}=\mathrm{O})$; ${ }^{1} \mathrm{H}-\mathrm{NMR}\left(\mathrm{DMSO}-d_{6}\right): \delta 3.18(\mathrm{dd}, 1 \mathrm{H}$, pyrazolyl-HA, $\left.J_{\mathrm{AX}}=3.6 \mathrm{~Hz}, J_{\mathrm{AB}}=16 \mathrm{~Hz}\right), 3.27\left(\mathrm{~s}, 3 \mathrm{H}, \mathrm{OCH}_{3}\right), 3.80\left(\mathrm{dd}, 1 \mathrm{H}\right.$, pyrazolyl-HB, $\left.J_{\mathrm{BX}}=12 \mathrm{~Hz}, J_{\mathrm{AB}}=16 \mathrm{~Hz}\right)$, $5.46\left(\mathrm{dd}, 1 \mathrm{H}\right.$, pyrazolyl-Hx, $\left.J_{\mathrm{AX}}=3.6 \mathrm{~Hz}, J_{\mathrm{BX}}=12 \mathrm{~Hz}\right), 6.83-7.51(\mathrm{~m}, 11 \mathrm{H}, \mathrm{Ar}-\mathrm{H}), 11.22(\mathrm{~s}, 1 \mathrm{H}, \mathrm{OH}$, $\mathrm{D}_{2} \mathrm{O}$ exchangeable); ${ }^{13} \mathrm{C}-\mathrm{NMR}$ (DMSO- $\left.d_{6}\right): \delta 40.3,66.8$ ( $\mathrm{C}_{3}$ and $\mathrm{C}_{4}$ dihydropyrazole), $56.3\left(\mathrm{OCH}_{3}\right)$, $110.6,116.1,118.7,123.5,124.8,129.1,130.8,137.1138 .3,146.0,146.5,148.5,150.2\left(\mathrm{C}_{6} \mathrm{H}_{3}, \mathrm{C}_{6} \mathrm{H}_{4}\right.$ 
and pyridine $\mathrm{C}), 164.3(\mathrm{C}=\mathrm{N})$ and $188.4(\mathrm{C}=\mathrm{O})$. Anal. Calcd. for $\mathrm{C}_{22} \mathrm{H}_{18} \mathrm{ClN}_{3} \mathrm{O}_{3}$ : $\mathrm{C}, 64.79 ; \mathrm{H}, 4.45 ; \mathrm{N}$, 10.30. Found: C, 64.69; H, 4.45; N, 10.29.

(3-(4-Bromophenyl)-5-(4-hydroxy-3-methoxyphenyl)-4,5-dihydro-1H-pyrazol-1-yl)(pyridin-4-yl) methanone (5d). The 4,5-dihydropyrazole was obtained in $86 \%$ yield; m.p. $168{ }^{\circ} \mathrm{C}$; IR $\left(\mathrm{KBr}, \mathrm{cm}^{-1}\right)$ : $3466(\mathrm{OH}), 1654(\mathrm{C}=\mathrm{N}), 1633(\mathrm{C}=\mathrm{O}) ;{ }^{1} \mathrm{H}-\mathrm{NMR}\left(\mathrm{DMSO}-d_{6}\right): \delta 3.20\left(\mathrm{dd}, 1 \mathrm{H}\right.$, pyrazolyl-H $\mathrm{H}_{\mathrm{A}}$, $\left.J_{\mathrm{AX}}=3.6 \mathrm{~Hz}, J_{\mathrm{AB}}=16 \mathrm{~Hz}\right), 3.22\left(\mathrm{~s}, 3 \mathrm{H}, \mathrm{OCH}_{3}\right), 3.77\left(\mathrm{dd}, 1 \mathrm{H}\right.$, pyrazolyl-HB, $\left.J_{\mathrm{BX}}=12 \mathrm{~Hz}, J_{\mathrm{AB}}=16 \mathrm{~Hz}\right)$, $5.47\left(\mathrm{dd}, 1 \mathrm{H}\right.$, pyrazolyl-Hx, $\left.J_{\mathrm{AX}}=3.6 \mathrm{~Hz}, J_{\mathrm{BX}}=12 \mathrm{~Hz}\right), 6.82-7.52(\mathrm{~m}, 11 \mathrm{H}, \mathrm{Ar}-\mathrm{H}), 11.20(\mathrm{~s}, 1 \mathrm{H}, \mathrm{OH}$, $\mathrm{D}_{2} \mathrm{O}$ exchangeable). Anal. Calcd. for $\mathrm{C}_{22} \mathrm{H}_{18} \mathrm{BrN}_{3} \mathrm{O}_{3}$ : C, 58.42; H, 4.01; N, 9.29. Found: C, 58.31; H, 3.89; N, 9.28.

\section{(5-(4-Hydroxy-3-methoxyphenyl)-3-(4-nitrophenyl)-4,5-dihydro-1H-pyrazol-1-yl)(pyridin-4-yl)}

methanone (5e). The 4,5-dihydropyrazole was obtained in $97 \%$ yield; m.p. $171{ }^{\circ} \mathrm{C}$; IR $\left(\mathrm{KBr}, \mathrm{cm}^{-1}\right)$ : $3471(\mathrm{OH}), 1649(\mathrm{C}=\mathrm{N}), 1634(\mathrm{C}=\mathrm{O}) ;{ }^{1} \mathrm{H}-\mathrm{NMR}$ (DMSO-d6): $\delta 3.17\left(\mathrm{dd}, 1 \mathrm{H}\right.$, pyrazolyl-H $\mathrm{H}_{\mathrm{A}}, J_{\mathrm{AX}}=3.6 \mathrm{~Hz}$, $\left.J_{\mathrm{AB}}=16 \mathrm{~Hz}\right), 3.39\left(\mathrm{~s}, 3 \mathrm{H}, \mathrm{OCH}_{3}\right), 3.69\left(\mathrm{dd}, 1 \mathrm{H}\right.$, pyrazolyl- $\left.\mathrm{H}_{\mathrm{B}}, J_{\mathrm{BX}}=12 \mathrm{~Hz}, J_{\mathrm{AB}}=16 \mathrm{~Hz}\right), 5.42$ $\left(\mathrm{dd}, 1 \mathrm{H}\right.$, pyrazolyl-Hx, $\left.J_{\mathrm{Ax}}=3.6 \mathrm{~Hz}, J_{\mathrm{BX}}=12 \mathrm{~Hz}\right), 6.84-7.71(\mathrm{~m}, 11 \mathrm{H}, \mathrm{Ar}-\mathrm{H}), 11.89(\mathrm{~s}, 1 \mathrm{H}, \mathrm{OH}$, $\mathrm{D}_{2} \mathrm{O}$ exchangeable). Anal. Calcd. for $\mathrm{C}_{22} \mathrm{H}_{18} \mathrm{~N}_{4} \mathrm{O}_{5}$ : C, 63.15; H, 4.34; N, 13.39. Found: C, 63.14; H, 4.28; N, 13.47 .

\subsection{Determination of Antimicrobial Activity}

All compounds were tested against four different microorganisms Staphylococcus aureus, Escherichia coli, Pseudomonas aeruginosa, Candida albicans. The agar well-diffusion method was applied for the determination of inhibition zone and minimum inhibitory concentration (MIC). Briefly, $0.75 \mathrm{~mL}$ of broth culture containing $\mathrm{ca} .10^{6}$ colony-forming units (CFU) per $\mathrm{mL}$ of the test strain was added to $75 \mathrm{~mL}$ of nutrient agar medium at $45^{\circ} \mathrm{C}$, mixed well, and then poured into a $15 \mathrm{~cm}$ sterile metallic Petri plate. The medium was allowed to solidify, and $8 \mathrm{~mm}$ wells were dug with a sterile metallic borer. Then, a DMSO solution of the test sample $(1 \mathrm{~mL})$ at $1 \mathrm{mg} / \mathrm{mL}$ was added to the respective wells. DMSO served as negative control, and the standard antimicrobial drugs Rifampicin (5 $\mu \mathrm{g} / \mathrm{disc})$ and Ampicillin $(10 \mu \mathrm{g} / \mathrm{disc})$ were used as positive controls. Triplicate plates for each microorganism strain were prepared and were incubated aerobically at $37{ }^{\circ} \mathrm{C}$ for $24 \mathrm{~h}$. The activity was determined by measuring the diameter of zone showing complete inhibition $(\mathrm{mm})$, thereby, the zones were precisely measured with the aid of a Vernier caliper (precision $0.1 \mathrm{~mm}$ ). The growth inhibition was calculated with reference to the positive control. For the individual compounds that showed inhibition zones $>10 \mathrm{~mm}$, MIC values were determined by means of the agar well-diffusion method for concentrations of $1.0,0.50,0.25,0.125,0.063$ and $0.031 \mathrm{mg} / \mathrm{mL}$ in DMSO. The tests were performed in triplicate, and the results were averaged. Also minimum bactericidal concentrations (MBC) were determined for all chloro derivatives which exhibited high activities (compounds 1c, 2c, 4c and 5c) for concentrations of $1.0,0.50,0.25,0.125,0.063$ and $0.031 \mathrm{mg} / \mathrm{mL}$ in DMSO. All our results are listed in Tables 1 and 2. 


\subsection{DPPH Based Free Radical Scavenging Activity}

Since DPPH is a stable free radical containing an odd electron in its structure, it is usually utilized for detection of the radical scavenging activity. Aliquots of different concentrations $(20-100 \mu \mathrm{g} / \mathrm{mL})$ of the test sample is added to $100 \mu \mathrm{L}$ solution DPPH (4 mg/100 mL methanol). Absorbance at $517 \mathrm{~nm}$ is determined after $30 \mathrm{~min}$. Each experiment was done in triplicate and average is taken. Vitamin $\mathrm{C}$ was used as a positive control and percentage of free radical scavenging was expressed as inhibition from the given formula:

$$
\% \text { inhibition of DPPH radical }=\frac{\text { Abs. of control }- \text { Abs. of sample }}{\text { Abs. of control }} \times 100
$$

Efficacy was calculated for $0.25 \mathrm{mg} / \mathrm{mL}$ of each compound by using the following equation:

$$
\text { Efficacy }=\frac{\text { DPPH scavenging } \% \text { of compound at } 0.25 \mathrm{~mL}}{\text { DPPH scavenging \% of vitamin } \mathrm{C} \text { at } 0.25 \mathrm{~mL}}
$$

Calculated antioxidant data of all the tested samples were summarized in Table 3.

\subsection{Nitric Oxide Radical Scavenging Activity}

Nitric oxide was generated from sodium nitroprusside and measured by Griess' reaction [62,63]. Reagents are sodium nitroprusside $(10 \mathrm{mM})$, phosphate buffer saline and Griess reagent $(1 \mathrm{~g}$ of sulphanilic acid $+0.1 \mathrm{~g}$ naphthylethylene diamine dihydrochloride). $20 \mu \mathrm{L}$ sodium nitroprusside, $5 \mu \mathrm{L}$ phosphate buffer and $5 \mu \mathrm{L}$ of compound were incubated at $25^{\circ} \mathrm{C}$ for $2.30 \mathrm{~h}$. After incubation, $20 \mu \mathrm{L}$ of griess reagent was added to the previous mixture and allowed to stand for $30 \mathrm{~min}$. The absorbance of the colour developed during diazotization of nitrite with sulphanilamide and its subsequent coupling with napthylethylenediamine hydrochloride was observed at $550 \mathrm{~nm}$ on spectrophotometer. Each experiment was done in triplicate and average is taken. Vitamin $\mathrm{C}$ was used as positive control and percentage of free radical scavenging was expressed as inhibition from the formula:

$$
\% \text { inhibition of NO radical }=\frac{\text { Abs. of control }- \text { Abs. of sample }}{\text { Abs. of control }} \times 100
$$

Efficacy was calculated for $0.25 \mathrm{mg} / \mathrm{mL}$ of each compound by using the following equations:

$$
\text { Efficacy }=\frac{\text { NO scavenging } \% \text { of compound at } 0.25 \mathrm{~mL}}{\text { NO scavenging } \% \text { of vitamin } \mathrm{C} \text { at } 0.25 \mathrm{~mL}}
$$

Calculated anti-inflammatory data of all the tested samples were summarized in (Table 4).

\section{Conclusions}

This work demonstrates a rapid, efficient method for the synthesis of new pyrazole and dihydropyrazole derivatives. All synthesized compounds were characterized by spectral data (IR, ${ }^{1} \mathrm{H}-\mathrm{NMR}$ and ${ }^{13} \mathrm{C}-\mathrm{NMR}$ ) and the structures were consistent with the data. All the synthesized compounds were tested for their antimicrobial activity against four test organisms. The results showed that the compounds that having pharmacophores with lipophilic properties such as chloro and bromo substituents exhibited the greatest 
antimicrobial activities. Also two pharmacological activities namely antioxidant and anti-inflammatory activity, were tested for the chloro derivatives $\mathbf{1 c}, \mathbf{2 c}, \mathbf{3 c}, \mathbf{4 c}, \mathbf{5 c}$. These activities vary according to their structures and functional groups.

\section{Acknowledgments}

The authors would like to acknowledge the help provided by Dooa Khareeb, Department of Biochemistry, Faculty of Science, Alexandria University, Alexandria, Egypt in carrying out the antioxidant and anti-inflammatory activity tests.

\section{Author Contributions}

Nagwa M. M. Hamada and Nadia Y. M. Abdo designed the research, performed the experiments and analyzed the data. Both authors contributed to the paper and approved the manuscript.

\section{Conflicts of Interest}

The authors declare no conflict of interest.

\section{References}

1. Taylor, E.C.; Patel, H.H. Synthesis of Pyrazolo [3,4-d] Pyrimidine Analogues of the potentagent. $N$-\{4-[2-(2-amino-4(3H)-oxo-7H-pyrrolo[2,3- $d$ ]Pyrimidin-5-yl) ethyl]benzoyl $\}$-L-Glutamic acid (LY231514). Tetrahedron 1992, 48, 8089-8100.

2. Song, H.; Liu, Y.; Xiong, L.; Li, Y.; Yang, N.; Wang, Q. Design, Synthesis and Insecticidal Activity of Novel Pyrazole Derivatives Containing $\alpha$-hydroxymethyl- $N$-Benzylcarboxamide, $\alpha$-Chloromethyl- $N$-Benzyl Carboxamide, and 4,5-Dihydrooxazole moieties. J. Agric. Food Chem. 2012, 60, 1470-1479.

3. Sharshira, E.M.; Hamada, N.M.M. Synthesis and Antimicrobial Evaluation of Some Pyrazole Derivatives. Molecules 2012, 17, 4962-4971.

4. Rashad, A.E.; Shamroukh, A.H.; Hegab, M.I.; Awad, H.M. Synthesis of Some Biologically Active Pyrazoles and C-Nucleosides. Acta Chim. Slov. 2005, 52, 429-434.

5. Rashad, A.E.; Hegab, M.I.; Abdel-Megeid, R.E.; Micky, J.A.; Abdel-Megeid, F.M.E. Synthesis and Antiviral Evaluation of Some New Pyrazole and Fused Pyrazolo Pyrimidine Derivatives. Bioorg. Med. Chem. 2008, 16, 7102-7106.

6. Bhat, B.A.; Dhar, K.L.; Saxena, A.K.; Shanmugavel, M.; Qazi, G.N. Synthesis and Biological Evaluation of Chalcones and Their Derived Pyrazoles as Potential Cytotoxic Agents. Bioorg. Med. Chem. Lett. 2005, 15, 3177-3180.

7. Horrocks, P.; Pickard, M.R.; Parekh, H.H.; Patel, S.P.; Pathak, R.B. Synthesis and Biological Evaluation of 3-(4-Chlorophenyl)-4-Substituted Pyrazole Derivatives. Org. Biomol. Chem. 2013, 11, 4891-4898.Kalirajan, R.; Sivakumar, S.U.; Jubie, S.; Gowramma, B.; Suresh, B. Synthesis and Biological Evaluation of Some Heterocyclic Derivatives of Chalcones. Int. J. ChemTech Res. 2009, 1, 27-34. 
9. Holla, B.S.; Akberali, P.M.; Shivanada, M.K. Studies on Arylfuran Derivative: Part X. Synthesis and Antibacterial Properties of Arylfuryl- $\Delta^{2}$-Pyrazolines. Farmaco 2000, 55, 256-263.

10. Maggio, B.; Daidone, G.; Raffa, D.; Plescia, S.; Mantione, L.; Cutuli, V.M.C.; Mangano, N.G.; Caruso, A. Synthesis and Pharmacological Study of ethyl 1-methyl-5-(substituted-3,4-dihydro-4oxoquinazolin-3-yl)-1H-pyrazole-4-acetates. Eur. J. Med. Chem. 2001, 36, 737-742.

11. Clinton, R.O.; Manson, A.J.; Stonner, F.W.; Beyler, A.L.; Potts, G.O.; Arnold, A. Steroidal [3,2-c] pyrazoles. J. Am. Chem. Soc. 1959, 81, 1513-1514.

12. Kalirajan, R.; Palanivelu, M.; Rajamanickam, V.; Vinothapooshan, G.; Andarajagopal, K. Synthesis and Biological Evaluation of Some Heterocyclic Derivatives of Chalcones. Int. J. Chem. Sci. 2007, 5, 73-80.

13. Urmila, G.; Vineeta, S.; Vineeta, K.; Sanjana, C. Synthesis and Antifungal Activity of New Fluorine Containing 4-(Substituted Phenylazo) Pyrazoles and Isoxazoles. Indian J. Heterocycl. Chem. 2005, 14, 265-266.

14. Patel, C.K.; Rami, C.S.; Panigrahi, B.; Patel, C.N. Synthesis and Biological Evaluation of (4-Substituted Benzylidene)-3-Methyl-1-(Substituted Phenyl Sulfonyl and Substituted Benzoyl)1H-Pyrazol-5(4H)-one As Anti-inflammatory Agent. J. Chem. Pharm. Res. 2010, 2, 73-78.

15. Yang, J.F.; Cao, H.; Liu, H.; Li, B.Q.; Ma, Y.M. Synthesis and Bioactivity of Novel Bis-heterocyclic Compounds Containing Pyrazole and Oxadiazoline. J. Chin. Chem. Soc. 2011, 58, 369-375.

16. MallikarjunaRao, R.; Sreeramulu, J.; Ravindranath, L.K.; NagarajaReddy, G.; Hanumanthurayudu, K.; Nageswara Reddy, G.; Jayaraju, A.; Madhusudhan, P. Synthesis and biological screening of some Pyridine and Pyrrole Derivatives of Pyrazolo [3,4-c] pyrazoles. J. Chem. Pharm. Res. 2012, 4, 272-278.

17. Mohareb, R.M.; El-Sayed, N.N.E.; Abdelaziz, M.A. Uses of Cyanoacetylhydrazine in Heterocyclic Synthesis: Novel Synthesis of Pyrazole Derivatives with Anti-tumor Activities. Molecules 2012, 17 , 8449-8463.

18. Kumar, K.A.; Jayaroopa, P. Pyrazoles: Synthetic Strategies and Their Pharmaceutical Applications-An Overview. Int. J. PharmTech Res. 2013, 5, 1473-1486.

19. Piste, P.B. Facile Synthesis and Antimicrobial Screening of Pyrazole Derivatives World. J. Pharm. Res. 2014, 3, 735-742.

20. Kamal, A.; Shaik, A.B.; Polepalli, S.; Reddy, V.S.; Kumar, G.B.; Gupta, S.; Krishna, K.V.S.R.; Nagabhushana, A.; Mishra R.K.; Jain, N. Pyrazole-Oxadiazole Conjugates: Synthesis, Antiproliferative Activity and Inhibition of Tubulin Polymerization. Org. Biomol. Chem. 2014, 12, 7993-8007.

21. Bhalla, A.; Bari, S.S.; Bhalla, J. Synthesis of Novel Pyrazolylmethylene-Pyrimidine Heterocycles: Potential Synthons for Hybrid $\beta$-Lactams. Can. Chem. Trans. 2015, 3, 72-84.

22. Abdelhamid, A.O.; Zohdi, H.F.; Sallam, M.M.M.; Ahmed, N.A. Reactions with Hydra- zonoyl Halides. 31. Synthesis of Some New Pyrrolidino [3,4-c] pyrazolines, Pyrazoles, and Pyrazolo [3,4- $d$ ] pyridazines. Molecules 2000, 5, 967-973.

23. Nakamichi, N.; Kawashita, Y.; Hayashi, M. Oxidative Aromatization of 1,3,5-Trisubstituted pyrazolines and Hantzsch 1,4-dihydropyridines by $\mathrm{Pd} / \mathrm{C}$ in acetic acid. Org. Lett. 2002, 4, 3955-3957. 
24. El-Emary, T.I. Synthesis of Newly Substituted Pyrazoles and Substituted Pyrazolo [3,4-b] pyridines Based on 5-Amino-3-methyl-1-phenyl pyrazole. J. Chin. Chem. Soc. 2007, 54, 507-518.

25. Hamada, N.M. Synthesis and Spectral Studies of Some Novel Pyrazole Derivatives from Chalcones Precursors. Heterocycl. Commun. 2009, 15, 327-334.

26. Hu, H.; Ge, C.; Ding, L.; Zhang, A. Synthesis of Novel 1-[(2,6-Dichloro-4-trifluoro-methyl) phenyl]-3-aryl-1H-pyrazole-4-carbaldehydes. Molecules 2010, 15, 7472-7481.

27. Sharshira, E.M.; Hamada, N.M.M. Synthesis and in Vitro Antimicrobial Activity of Some Pyrazolyl-1-carboxamide Derivatives. Molecules 2011, 16, 7736-7745.

28. Ghorab, M.M.; Ragab, F.A.; Heiba, H.I.; Agha, H.M. Synthesis of Some Novel Sulfonamides Containing Biologically Active Alkanoic Acid, Acetamide, Thiazole, and Pyrrole Moieties of Expected Antitumor and Radiosensitizing Activities. J. Basic Appl. Chem. 2011, 1, 8-14.

29. Fadda, A.A.; Abdel-Latif, E.; El-Mekawy, R.E. Synthesis of Some New Aryl Azo Thiophene and Arylazopyrazole Derivatives as Antitumor Agents. Pharmacol. Pharm. 2012, 3, 148-157.

30. Ghorab, M.M.; El-Gazzar, M.G.; Alsaid, M.S. Synthesis, Characterization and Anti-Breast Cancer Activity of New 4-Aminoantipyrine-Based Heterocycles. Int. J. Mol. Sci. 2014, 15, 7539-7553.

31. Penning, T.D.; Talley, J.J.; Bertenshawm, S.R.; Carter, J.S.; Collins, P.W.; Docter, S.; Graneto, M.J.; Lee, L.F.; Malecha, J.W.; Miyashiro, J.M.; et al. Synthesis and Biological Evaluation of the 1,5-Diarylpyrazole Class of Cyclooxygenase-2 Inhibitors: Identification of 4-[5-(4-methylphenyl)3-(trifluoromethyl)-1H-pyrazole-1-yl]benzenesulfonamide. J. Med. Chem. 1997, 40, 1347-1365.

32. Korgaokar, S.S.; Patil, P.H.; Shah, M.J.; Parekh, H.H. Studies on Pyrazolines: Preparation and Antimicrobial Activity of 3-(3-(p-Chlorophenylsulphonamidophenyl)-5Aryl-1H/Acetyl Pyrazolines. Ind. J. Pharm. Sci. 1996, 58, 222-225.

33. Amir, M.; Kumar, H.; Khan, S.A. Synthesis and Pharmacological Evaluation of Pyrazoline Derivatives as New Anti-Inflammatory and Analgesic Agents. Bioorg. Med. Chem. Lett. 2008, 18, 918-922.

34. Sridhar, S.; Rajendraprasad, Y. Synthesis and analgesic studies of some new 2-pyrazolines. J. Chem. 2012, 9, 1810-1815.

35. Palaska, E.; Erol, D.; Demirdamar, R. Synthesis and antidepressant activities of some 1,3,5-triphenyl-2-pyrazolines. Eur. J. Med. Chem. 1996, 31, 43-47.

36. Palaska, E.; Aytemir, M.; Uzbay, I.T.; Erol, D. Synthesis and Antidepressant Activities of some 3,5-Diphenyl-2-Pyrazolines. Eur. J. Med. Chem. 2001, 36, 539-543.

37. Gok, S.; Demet, M.M.; Özdemir, A.; Turan-Zitouni, G. Evaluation of Antidepressant-Like Effect of 2-Pyrazoline Derivatives. Med. Chem. Res. 2010, 19, 94-101.

38. Dmytro, H.; Borys, Z.; Olexandr, V.; Lucjusz, Z.; Andrzej, G.; Roman, L. Synthesis of Novel Thiazolone-Based Compounds Containing Pyrazoline Moiety and Evaluation of Their Anticancer Activity. Eur. J. Med. Chem. 2009, 44, 1396-1404.

39. Raman, K.; Pandey, B.R.; Barthwal, J.P.; Parmar, S.S. Antiinflammatory and Antiproteolytic Properties of 1,3-Disubstituted-5-(2-arylindol-3-yl)- $\Delta^{2}$-pyrazolines. Eur. J. Med. Chem. Chim. Ther. 1980, 15, 567-569.

40. Mui, M.S.; Siew, B.N.; Buss, A.D.; Crasta, S.C.; Kah, L.G.; Sue, K.L. Synthesis of N-1 Acidic Functionality Affording Analogues With Enhanced Antiviral Activity Against HIV. Bioorg. Med. Chem. Lett. 2002, 12, 679-699. 
41. Turan-Zitounim, G.; Chevallet, P.; Kiliç, F.S.; Erol, K. Synthesis of Some Thiazolyl-Pyrazoline Derivatives and Preliminary Investigation of Their Hypotensive Activity. Eur. J. Med. Chem. 2000, 35, 635-641.

42. Ahmad, A.; Husain, A.; Khan, S.A.; Mujeeb, M.; Bhandari, A. Synthesis, Antimicrobial and Antitubercular Activities of Some Novel Pyrazoline Derivatives. J. Saudi Chem. Soc. 2014, doi:10.1016/j.jscs.2014.12.004.

43. Soni, N.; Pande, K.; Kalsi, R.; Gupta, T.K.; Parmar, S.S.; Barthwal, J.P. Inhibition of Rat Brain Monoamine Oxidase and Succinic Dehydrogenase by Anticonvulsant Pyrazolines. Res. Commun. Mol. Pathol. Pharm. 1987, 56, 129-132.

44. Rairord, L.C.; Gundy, G.V. Condensation of Vanillin Substitution Products with Aceto- phenone. J. Am. Chem. Soc. 1932, 54, 1191-1193.

45. Modzelewska, A.; Pettit, C.; Achanta, G.; Davidson, N.E.; Huang, P.; Khan, S.R. Anticancer activities of novel chalcone and bis-chalcone derivatives. Bioorg. Med. Chem. 2006, 14, 3491-3495.

46. Ansari, F.L.; Nazir, S.; Noureen, H.; Miraza, B. Combinatorial Synthesis and Antibacterial Evaluation of an Indexed Chalcone Library. Chem. Biodivers. 2005, 2, 1656-1664.

47. Sadhu, S.K.; Okuyama, E.; Fujimoto, H.; Ishibashi, M. Separation of Leucas Aspera, a Medicinal Plant of Bangladesh, Guided by Prostaglandin Inhibitory and Antioxidant Activities. Chem. Pharm. Bull. 2003, 51, 595-598.

48. Brand-Willams, W.; Cuvelier, M.E.; Berset, C. Use of a Free Radical Method to Evaluate Antioxidant Activity. Lebensm. Wiss. Technol. 1995, 28, 25-30.

49. Espin, J.C.; Soler-Rivas, C.; Wichers, H.J. Characterization of Total Free Radical Scavenger Capacity of Vegetable Oils and Oil Fraction Using 2,2-Diphenyl-1-picrylhydrazyl Radical. J. Agric. Food. Chem. 2000, 48, 648-656.

50. Yu, L. Free Radical Scavenging Properties of Conjugated Linoleic Acids. J. Agric. Food Chem. 2001, 49, 3452-3456.

51. Sherwin, E.R. Oxidation and Antioxidants in Fat and Oil Processing. J. Am. Oil Chem. Soc. 1978, 55, 809-841.

52. Hossain, M.M.; Shaha, S.K.; Foysal Aziz, F. Antioxidant Potential Study of Some Synthesized N-Heterocycles. Bangladesh Med. Res. Counc. Bull. 2009, 35, 49-52.

53. Herbert, V. Prooxidant Effects of Antioxidant Vitamins. J. Nutr. 1996, 126, 1197S-1200S.

54. Fukumotoand, L.R.; Mazza, G. Assessing Antioxidant and Prooxidant Activities of Phenolic Compounds. J. Agric. Food Chem. 2000, 48, 3597-3604.

55. Hagerman, A.E.; Riedl, K.M.; Jones, G.A.; Sovik, K.N.; Ritchard, N.T.; Hartzfeld, P.W. High Molecular Weight Plant Polyphenolics (tannins) as Biological Antioxidants. J. Agric. Food Chem. 1998, 46, 1887-1892.

56. Nabavi, S.M.; Ebrahimzadeh, M.A.; Nabavi, S.F.; Hamidinia, A.; Bekhradnia, A.R. Determination of Antioxidant Activity, Phenol and Flavonoids Content of Parrotia Persica Mey. Pharmacol. Online 2008, 2, 560-567.

57. Nabavi, S.M.; Ebrahimzadeh, M.A.; Nabavi, S.F.; Jafari, M. Free Radical Scavenging Activity and Antioxidant Capacity of Eryngium Caucasicum Trautv and Froripia Subpinnata. Pharmacol. Online 2008, 3, 19-25. 
58. Gulati, K.; Ray, A.; Masood, A.; Vijayan, V.K. Involvement of Nitric Oxide (NO) in the Regulation of Stress Susceptibility and Adaptation in Rats. Ind. J. Exp. Biol. 2006, 44, 809-815.

59. Krishnakumar, K.; Bhat, A.R.; Umaa, K.; Chandrasekharan A.K. Synthesis and Valuation of Mannich Bases of Certain Novel Nitro Hydroxy 1,2-Pyrazolines. Anc. Sci. Life 2004, 24, 103-108.

60. Mounika, S. Synthesis Characterization and Evaluation of Some Pyrazoline Derivatives. Int. J. Innov. Pharm. Sci. Res. 2013, 1, 235-251.

61. Sharma, N.; Mohanakrishnanb, D.; Sharma, U.K.; Kumar, R.; Richa Sinha, A.K.; Sahal, D. Design, Economical Synthesis and Antiplasmodial Evaluation of Vanillin Derived Allylated Chalcones and Their Marked Synergism with Artemisinin against Chloroquine Resistant Strains of Plasmodium falciparum. Eur. J. Med. Chem. 2014, 79, 350-368.

62. Marcocci, L.; Maguire, J.; Droy-Lefaix, M.T.; Packer, L. The Nitric Oxide Scavenging Properties of Ginkgo Biloba Extract EGB 761. Biochem. Biophys. Res. Commun. 1994, 201, 748-755.

63. Sreejayan, N.; Rao, M.N.A. Nitric Oxide Scavenging by Curcuminoids. J. Pharm. Pharmacol. 1997, 49, 105-107.

Sample Availability: Samples of the compounds $\mathbf{1 a}-\mathbf{e}, \mathbf{3 c}, \mathbf{d}, \mathbf{4 b}-\mathbf{d}$ and $\mathbf{5 c}, \mathbf{d}$ are available from the authors.

(C) 2015 by the authors; licensee MDPI, Basel, Switzerland. This article is an open access article distributed under the terms and conditions of the Creative Commons Attribution license (http://creativecommons.org/licenses/by/4.0/). 